\title{
Transcriptome Dynamics Reveal Stage-specific and Melatonin-triggered Gene Expression Patterns During the Cashmere Growth Cycle in Capra Hircus
}

\section{Chun Li}

Inner Mongolia University for Nationalities

\section{Cong Feng}

Zhejiang University College of Life Sciences

\section{Guangyuan Ma}

Zhejiang University College of Life Sciences

\section{Shaoyin Fu}

Inner Mongolia Academy of Agricultural and Animal Husbandry Sciences

Ming Chen ( $\nabla$ mchen@zju.edu.cn )

Zhejiang University College of Life Sciences

\section{Wenguang Zhang}

Inner Mongolia Agricultural University

Jinquan Li

Inner Mongolia Agricultural University https://orcid.org/0000-0002-8616-668X

\section{Research}

Keywords: cashmere growth cycle, hair follicle, RNA-seq, melatonin, signaling pathway

Posted Date: June 9th, 2021

DOl: https://doi.org/10.21203/rs.3.rs-586481/v1

License: (c) (i) This work is licensed under a Creative Commons Attribution 4.0 International License.

Read Full License 


\title{
Transcriptome Dynamics Reveal Stage-specific and Melatonin-triggered Gene Expression Patterns during the Cashmere Growth Cycle in Capra hircus
}

\author{
Chun $\mathrm{Li}^{1 \dagger}$, Cong Feng ${ }^{2 \dagger}$, Guangyuan $\mathrm{Ma}^{2 \dagger}$, Shaoyin $\mathrm{Fu}^{3}$, Ming Chen ${ }^{2,4^{*}}$, WenguangZhang ${ }^{5 *}$ and Jinquan $\mathrm{Li}^{5^{*}}$
}

\author{
${ }^{*}$ Correspondence: \\ mchen@zju.edu.cn; \\ atcgnmbi@yahoo.com.cn \\ lijinquan_nd@126.com \\ ${ }^{2}$ College of Life Sciences, Zhejiang \\ University, Hangzhou 310058 \\ China \\ ${ }^{4}$ College of Life Science and Food \\ Engineering, Inner Mongolia \\ University for Nationalities \\ Tongliao 028000, China \\ ${ }^{5}$ College of Animal Science, Inner \\ Mongolia Agricultural University, \\ Hohhot 010018, China \\ $\dagger$ These authors have contributed \\ equally to this work. \\ Full list of author information is \\ available at the end of the article
}

\begin{abstract}
Background: Cashmere goat is famous for its high-quality fibers. The growth of cashmere in secondary hair follicles exhibits a seasonal pattern arising from circannual changes in the natural photoperiod. Although several studies have compared and analyzed the differences in gene expression between different cashmere growth stages, the selection of samples in these studies relies on research experience or morphological evidence. Distinguishing cashmere growth cycles according to gene expression patterns mayhelp to exploretheregulation mechanisms related to cashmere growth and the effect of melatonin from a molecular level more accurately.
\end{abstract}

Results: In this study, we applied RNA-sequencing to the hair follicles of three normal and three melatonin-treated Inner Mongolian cashmere goats sampled every month during a whole cashmere growth cycle. A total of 3559 and 988 genes were subjected as seasonal changing genes (SCGs) in the control and treated groups, respectively. The SCGs in the normal group are divided into three clusters, and their specific expression patterns help to group the cashmere growth cycle into anagen, catagen and telogen stages. Some canonical pathways such as Wnt, TGF-beta and Hippo signaling pathways are detected as promoting the cashmere growth, while Cell adhesion molecules (CAMs), Cytokine-cytokine receptor interaction, Jak-STAT, Fc epsilon RI, NOD-like receptor, Rap1, PI3K-Akt, cAMP,NF-kappaB andmany immune-related pathways are detected in the catagen and telogen stages. The PI3K-Akt signaling, ECM-receptor interactionand Focal adhesion are found in the transition stage between telogen to anagen, which may serve as candidate biomarkers for telogen-anagen regeneration. Pairwise comparisons between the control and melatonin-treated groups also indicate 941 monthly differentially expressed genes (monthly DEGs). These monthly DEGs are mainly distributed from April and September, which reveal a potential signal pathway map regulating the anagen stage triggered by melatonin. Enrichment analysis shows that Wnt, Hedgehog, ECM, Chemokines and NF-kappa B signaling pathways may be involved in the regulation of non-quiescence and secondary shedding under the influence of melatonin.

Conclusions: Our study decodes the key regulators of the whole cashmere growth cycle, laying the foundation for the control of cashmere growth and improvement of cashmere yield.

Keywords: cashmere growth cycle; hair follicle; RNA-seq; melatonin; signaling pathway 


\section{Background}

2 The Inner Mongolian cashmere goat (Capra hircus), an excellent cashmere goat breed

3 in China, is famous for producing cashmere with superior quality and high yield. Inner

4 Mongolian cashmere goats have two distinctly different fibrous hair structures, with

5 thick, coarse guard hairs forming the outer layer and fine, soft ground hairs forming the

6 cashmere underneath. The cashmere comes from secondary hair follicles (SHFs) in the

7 skin [1], and the coarse hair comes from primary hair follicles [2, 3]. The cashmere

8 obtained from goats is used exclusively in the production of luxurious textile products

9 [4]. The growth of cashmere in the Inner Mongolian cashmere goat exhibits a seasonal

10 pattern arising from circannual changes in the natural photoperiod. The cashmere

11 growth cycle typically starts in July and stopping the following March with shedding

12 of the fleece at the end of April [5].

13 Melatonin is a critical intermediary between photoperiod and cashmere growth, and

14 circulating melatonin levels directly affect cashmere growth [6]. Previous studies have

15 shown that the use of exogenous melatonin could stimulate cashmere growth during the

16 resting period. However, the implanting time and duration of melatonin could result in

17 earlier cashmere shedding followed by another typical cycle of cashmere growth [6-9],

18 or increase cashmere yield by combining the induced growth of cashmere fleece [10,

19 11]. It has been confirmed that exogenous melatonin has a positive role on cashmere

20 growth. Unfortunately, previous experiments have not been able to show the cashmere 
21 growth cycle related gene expression profiles and the potential roles of exogenous melatonin on cashmere growth.

23 Hair follicle growth in the skin of cashmere goats changes dynamically after birth and

24 continues in a cyclical pattern. The cashmere growth cycle can be divided into three 25 phases: telogen, anagen, and catagen, each of which is regulated by specific genetic patterns [5]. With the rapid development of high-throughput sequencing technology,

27 some regulatory factors and signaling pathways involved in the hair follicle cycle have 28 been found through differential expression and functional enrichment analysis. These 29 well-known regulatory molecules and signaling pathways include $\mathrm{Wnt} / \beta$ - catenin [12-

30 14], bone morphogenetic proteins (BMPs) [15, 16], sonic hedgehog (SHH) [17], notch

31 [18], fibroblast growth factors (FGFs) [19], transforming growth factors (TGFs) [20] 32 and keratin-associated proteins (KRTAPs) [5, 21], etc.

33 Long noncoding RNAs (lncRNAs) are RNAs that are longer than 200 nucleotides but 34 do not encode proteins. However, IncRNAs can regulate the expression of protein35 coding genes at various levels, including epigenetic regulation, transcriptional regulation and posttranscriptional regulation, thereby influencing various biological processes. Recent studies have reported the mechanisms of lncRNAs in hair growth and development in cashmere goats. For example, several important hair follicle 39 development signals (lncRNAs and mRNAs) are involved in primary wool follicle induction in carpet sheep [22]. Yin et al. indicated that lncRNA-599554 contributes the

41 inductive property of dermal papilla cells in cashmere goat, which might be achieved 
through sponging chi-miR-15b-5p to promote the WNT3A expression [23]. Wang et al. integrated analysis of lncRNA, miRNA and mRNA in cashmere goat skin during anagen and telogen stages and revealed potential ceRNA regulatory networks $[24,25]$.

Sulayman et al. performed a comprehensive analysis of lncRNA and mRNA expression profiles during sheep fetal and postnatal hair follicle development and demonstrated that the interaction between lncRNA and their target genes may regulate the development of hair follicles [26]. However, the roles of lncRNAs in controlling the whole cashmere growth cycle have not been well described.

To clarify the regulatory mechanism of cashmere growth cycles and gain insight into the gene regulatory network perturbed by exogenous melatonin, we use RNA-seq analysis to investigate the expression patterns of seasonal changing genes (SCGs) among different gene clusters. The interactions between lncRNAs and mRNAs were also explored using co-expression network analysis. The monthly differentially expressed genes obtained from pairwise comparisons between the control and melatonin-treated groups were detected to identify the key regulators associated with the growth of secondary hair follicles and reveal potential signaling pathways which may be involved in melatonin-affected growth patterns. 


\section{Methods}

\section{Skin sample and cashmere collection}

We enrolled six cashmere goats of the same gender and age. All goats were randomly divided into two groups: control group (D) and melatonin group (M). Three cashmere goats in the experimental group were subcutaneously implanted with melatonin for one year since December 2014. The control group was untreated. The skin samples from the six cashmere goats were collected monthly (Jan $19^{\text {th }}$, Feb $13^{\text {th }}$, Mar $21^{\text {st }}$, Apr $18^{\text {th }}$, May $20^{\text {th }}$, Jun $20^{\text {th }}$, Jul $18^{\text {th }}$, Aug $23^{\text {rd }}$, Sept $19^{\text {th }}$, Oct $20^{\text {th }}$, Nov $20^{\text {th }}$, and Dec $20^{\text {th }}$ ) from both the control and melatonin groups. Immediately after collection, all samples were frozen in liquid nitrogen and stored at $-80^{\circ} \mathrm{C}$ until use. At each sampling time point, 50 cashmeres were taken from each goat to measure the cashmere length.

\section{RNA extraction, library preparation and sequencing}

Total RNA from 72 collected skin samples were isolated using the TRIzol ${ }^{\mathrm{TM}}$ reagent (Invitrogen, USA) following the manufacturer's instructions. RNA degradation and contamination was monitored on $1 \%$ agarose gels. RNA purity was verified using the NanoPhotometer ${ }^{\circledR}$ spectrophotometer (Implen, USA). RNA integrity was checked using the RNA Nano 6000 Assay Kit (Agilent Technologies, USA). For the RNA library preparation, ribosomal RNA (rRNA) was removed first by the Epicentre Ribozero $^{\text {TM }}$ rRNA Removal Kit (Epicentre, USA) from $3 \mu \mathrm{g}$ total RNA. Then, sequencing libraries were generated using the NEBNext ${ }^{\circledR}$ Ultra $^{\mathrm{TM}}$ Directional RNA Library Prep Kit for Illumina (NEB, USA) following manufacturer's instructions. Finally, the strand- 
86 bp reads).

\section{Quality control}

88 FastQC [27] is used to filter out low-quality residues and joints of raw reads produced

89 by RNA-seq. The quality of clean reads (Q20, Q30, and GC content) were detected.

90 The high quality clean reads are retained for subsequent analysis.

\section{Read alignment and transcriptome assembly}

92 Clean reads were aligned to the goat genome (NCBI assembly ARS1) using HISAT2

93 [28] under default settings. The mapped reads of each sample were assembled by

94 StringTie [28] in a reference-based mode. All transcripts assembled were merged into

95 a Gene transfer format (GTF) file.

96 Novel IncRNA identification

97 The transcripts without matching the known annotations were selected to identify novel 98 lncRNAs through the following steps: (1) transcripts less than $200 \mathrm{bp}$ in length were

99 removed; (2) transcripts with exon number less than 2 were removed; (3) the coding

100 potential of each transcript was predicted using CPC2 [29] and CNCI [30], and

101 transcripts predicted as "noncoding" were imported into Pfam Scan

102 (http://ftp.ebi.ac.uk/pub/databases/Pfam/Tools/) to filter out those with known protein

103 family domains (Pfam release 30).

104 Reads counting and time-series differential expression analysis

105 All coding genes and lncRNA genes were included to calculate the count matrix using 106 htseq-count [31]. The likelihood ratio test (LRT) in DESeq2 [32] was used to identify

107 the seasonal changing genes (SCGs) in both control and melatonin group with model 
108 “ time" versus “ 1". Here SCGs are defined as genes with significant expression

109 changes throughout the cashmere growth cycle. Initially, the count matrix was

110 normalized by the size factors estimated by DESeq2 and the median of the normalized

111 gene expression values at each time point were used to calculate the fold change $f_{i}$ :

114 Where $G_{i j t}$ is the normalized expression value of gene $i$ in goat $j$ at sampling time $t$.

115 The thresholds for adjusted p-value and fold change were set to 0.05 and 2, respectively.

116 Besides, the differential changing genes (DCGs) between control and melatonin group

117 were detected using LRT with model " time + condition + time: condition" versus "

118 time + condition".

\section{Gene cluster detection}

120 The SCGs detected in control group were included for weighted gene co-expression

121 network analysis (WGCNA) [33]. Pearson correlations between gene clusters and trait 122 data (months) were calculated. The minimum cluster size was set to 50, and clusters

123 with a tree height less than 0.25 in the dendrogram were merged.

\section{Pathway enrichment}

125 The DAVID database [34] was used to identify the significantly enriched Kyoto

126 Encyclopedia of Genes and Genomes (KEGG) pathways [35] for gene modules

127 detected by WGCNA. The p-value threshold for enrichment was set to 0.05 . The 128 pathways enriched in infectious diseases and cancer were removed.

129 Monthly DEGs were annotated with Gene Ontology (GO) [36] biological process (BP) 
130 and KEGG analysis. The annotations were all achieved with clusterProfiler [37]

131 package, with $\mathrm{q}$-value $\leq 0.05$ for $\mathrm{GO}$ and $\mathrm{p}$-value $\leq 0.05$ for KEGG. Annotation

132 information was retrieved from the Ensembl database using AnnotationHub [38] to

133 generate an OrgDb annotation file.

\section{Pathway-mRNA-IncRNA network construction}

135 We selected genes enriched in key signaling pathways as sources, and co-expressed

136 lncRNA genes as targets to construct the pathway-mRNA-lncRNA network. The

137 Pearson correlations between pathway genes and lncRNA genes were calculated, and

138 those mRNA-IncRNA pairs with correlation higher than 0.8 were selected to construct

139 the network. CytoScape [39] was used to visualize the network.

140 Calculation of relative gene expression for monthly DEGs

141 To observe the effects of melatonin on the expression of representative differentially

142 expressed gene, we plotted relative expression boxplots to visually compare the

143 expression trends of these genes. The horizontal axis of the boxplot represents months,

144 and the vertical axis represents relative expression levels, which were calculated as 145 follows:

$$
\text { relative expression level }=\ln \left(\frac{\text { Gene Counts in Melatonin }}{\text { Gene Counts in Control }}\right)
$$

147 The gene counts were normalized with total counts in different groups. Three relative

148 expression values per month were used for boxplot visualization. 


\section{Results}

\section{Transcriptome analysis and differential gene expression overview}

154 A total of 72 samples spanning 12 months were analyzed in the control (D) and

155 melatonin (M) groups (Figure 1A). The experiments produced 2,959,842,480 clean

156 reads in total $(\sim 926 \mathrm{G})$. A total of 22,404 genes were detected from reads counting

157 through the RNA-seq data analysis (Additional file 1: Table S1). A total of 2365 novel

158 lncRNA genes were identified after the protein-coding-potential test (Additional file 2:

159 Table S2). Using DESeq2 time-series data analysis, 3559 and 988 genes were subjected

160 as SCGs by fold change $\geq 2$ and adjusted $\mathrm{p}$-value $\leq 0.05$ in the $\mathrm{D}$ and $\mathrm{M}$ groups,

161 respectively (Figure 1B) (Additional file 3: Table S3 and Additional file 4: Table

162 S4). Among these SCGs, a total of 345 novel lncRNAs and 211 annotated lncRNAs

163 were detected in the D group. We found that SCGs in the M group were much less than

164 those in the D group, and 76.8\% (759) of the SCGs in the M group also existed in the

165 D group (Figure 1C). According to the PCA diagrams in Figures 1D and 1E, the

166 samples of the D group showed a certain periodic distribution, but the sample

167 distribution was disturbed after the melatonin treatment. It can be seen in Figure 1F

168 and $\mathbf{1 G}$ that the goats in two groups shed the cashmere in May, while goats in the M

169 group had another shedding in September-November. An interesting finding is that in

170 the M group, new cashmere has grown during the shedding in May, that is, the SHFs

171 have not entered the resting period and the cashmere growth cycle has restarted in

172 advance (Figure 1G). 
173 Stage-specific gene expression dynamics in the cashmere growth cycle

174 The correct division of the cashmere growth cycle is the basis for subsequent

175 identification and analysis of regulatory factors. The existing studies are mostly based

176 on morphological evidence or prior knowledge. A recent study divided the cashmere

177 growth cycle into growth period, regression period and resting period based on skin

178 tissue sections and transcriptome data [5]. However, their analysis is based on static

179 differential expression analysis, the dynamic change of gene expression has not been

180 well studied. In our study, we analyzed the time-series transcriptome data covering the

181 entire cashmere growth cycle and obtained 3559 SCGs. Through WGCNA analysis,

182 three key gene clusters (DC1, DC2 and DC3) that may be involved in the growth cycle

183 of villi were identified in the D group (Figure 2A). The detailed genes of three clusters

184 are listed in Additional file 5: Table S5, which encompass 48.2\% (1717) of the SCGs

185 detected in the D group, including 91 annotated lncRNAs and 131 novel lncRNAs

186 (Figure 2B). Combining the expression patterns and functional enrichment results of

187 three clusters (Figure 2A and 2C), we inferred the effects of these clustered genes on

188 the cashmere growth cycle. The genes in DC1 may promote cashmere growth and the

189 genes in DC2 may be related to the regression of the SHFs. There may be an

190 antagonistic relationship between DC1 and DC2, which can also be inferred from the

191 gene expression trends in Figure 2D. The gene expression in DC1 began to be up-

192 regulated in April and reached a peak in October and started to be down-regulated,

193 while the gene expression pattern in DC2 was exactly the opposite. In addition, it can

194 also be found that the high expression of DC3 occurs when the expression of DC1 genes 
195 begins to be up-regulated and the expression of DC2 genes begins to down-regulate.

196 Based on the above analysis, we divided the cashmere growth cycle into three stages

197 associated with three gene clusters: (1) anagen (April-October); (2) catagen and telogen

198 (October-December and January-April); (3) telogen-anagen regeneration (February-

199 May). The detailed functional analysis of these three clusters and their relationships

200 with the cashmere growth cycle are discussed below.

201 The DC1 cluster shows high expression in anagen progression stage

202 A total of 394 genes in DC1 (including 22 annotated lncRNAs and 52 novel lncRNAs)

203 are positively correlated with the anagen progression stage (April-October), which may

204 be involved in promoting the cashmere growth (Figure 2A and Figure 2D). Some

205 canonical pathways such as the Wnt, TGF-beta and Hippo signaling pathways, which

206 have been proven to be closely related to hair growth [14, 40, 41] are also enriched in

207 DC1 (Figure 2C) (Additional file 6: Table S6). Three genes of the Wnt family

208 WNT2/WNT2B/WNT11, together with their receptors FZD3 and FZD10 are involved

209 in the regulation of the SHF development in cashmere goats. Lymphoid enhancer

210 binding factor-1 (LEF1) is an essential transcription factor in the Wnt signaling, and it

211 was strongly expressed during the anagen progression stage in this study. Its function

212 in hair cell differentiation and follicle morphogenesis has already been discussed [42].

213 Genes in the TGF-beta signaling (BMP2, BMP8A, BAMBI and SMAD6) also showed

214 similar patterns with the Wnt family. It is found that the genes enriched in the Hippo

215 signaling overlap with those in the Wnt and TGF-beta signaling, indicating the crosstalk

216 between Wnt and TGF-beta signaling formed by the joint regulation of downstream 
217 pathways [14, 43]. In addition, some downstream regulatory mechanisms of hair

218 growth and cycling were also confirmed in this study. For example, the homeobox

219 transcription factor DLX3 and one of its regulating genes, HOXC13, were also highly

220 expressed in the anagen stage. The regulatory cascade positions DLX3 downstream of

221 Wnt signaling and regulates other transcription factors related to hair follicle (HF)

222 differentiation (such as HOXC13) [44]. KRTs (KRT26, KRT35, KRT36, KRT39,

223 KRT6A, KRT74 and KRT84) and KRTAPs (KRTAP3-1 and KRTAP11-1) in DC1

224 were also associated with HF development [45]. SHH and its receptor $\mathrm{PTCH} 2$ in the 225 sonic hedgehog (Shh) signaling pathway were also found in DC1. The function of Shh 226 signaling is indicated as an essential regulator for controlling ingrowth and 227 morphogenesis of the HFs [46-48], but it is not necessary for initiating the HF 228 development [46].

229 The DC2 cluster prefers to be highly expressed in catagen and telogen

230 A total of 919 genes in DC2 (including 45 annotated lncRNAs and 53 novel lncRNAs)

231 were up-regulated from October to April of the following year (Figure 2A and 2D),

232 which corresponded to the degenerative and resting periods of the cashmere growth 233 cycle. It should be pointed out that due to the limitation of sampling interval, the catagen 234 and telogen stages were not further distinguished in this study. The expression pattern 235 of DC2 is negatively correlated with DC1, suggesting that there may be an antagonistic 236 relationship between the genes in DC2 and DC1. DC2 genes are mainly enriched in 237 pathways such as Cell adhesion molecules (CAMs), Cytokine-cytokine receptor 238 interaction, Jak-STAT, Fc epsilon RI, NOD-like receptor, Rap1, PI3K-Akt, cAMP, NF- 
239 kappa B and many immune-related pathways (Figure 2C) (Additional file 6: Table

240 S6). A previous study also found that differentially expressed genes between anagen

241 and telogen SHF-derived dermal papilla cells of the Cashmere goat were also enriched

242 in CAMs and Cytokine-cytokine receptor interaction pathways [49]. A number of

243 interleukin (IL) superfamily genes were involved in the enriched pathways of DC2,

244 such as the IL1 family (IL7 and IL18), IL10, IL15, and IL receptors (IL2RG, IL3RA,

245 IL6R, IL7R, IL11RA and IL20RA). The CC chemokine subfamily (CCL5, CCL21,

246 CCL22 and CCL26) and receptors (CCR4 and CCR6) were also found in the DC2

247 cluster. These chemokines are mainly involved in cell migration, immunity and

248 inflammation [50]. During the HF regression, these chemokines may guide the

249 migration of immune cells such as dendritic cells [51] and Regulatory $\mathrm{T}$ cells [52],

250 thereby regulating the immune response to apoptotic cells. The JAK3 and STAT4 genes

251 in the JAK-STAT signaling were highly expressed in catagen and telogen, which have

252 been found to maintain HF stem cell quiescence and inhibit hair growth [53-55]. In

253 addition, Dickkopf1 (DKK1), a Wnt signaling inhibitor, was also found in DC2. DKK1

254 has been strongly suggested to promote regression of HFs by suppressing Wnt/ $\beta$ -

255 catenin signaling and inducing apoptosis in follicular keratinocytes $[56,57]$.

256 The DC3 cluster is specifically expressed in telogen-anagen regeneration stage

257 DC3 cluster contains a total of 404 genes (including 24 annotated lncRNAs and 26

258 novel lncRNAs) and shows specific high expression during the transition period from

259 February to May (Figure 2A and 2D). Due to the overlap of the early anagen and

260 telogen-anagen regeneration stages, the genes of DC3 and DC2 were partially enriched 
261 in several same pathways like CAMs, Focal adhesion, extracellular matrix (ECM)262 receptor interaction, PI3K-Akt and NF-kappa B signaling (Figure 2C) (Additional file

263 6: Table S6). The PI3K-Akt signaling has been proved to be essential for HF 264 regeneration $[58,59]$. A previous study found that the Toll-Like Receptor 3 (TLR3) 265 activated by a dsRNA was able to promote HF regeneration [60]. Several collagen genes (COL1A1, COL1A2, COL3A1, COL5A2, COL6A3, COL6A5 and COL6A6) in

267 the PI3K-Akt signaling, ECM-receptor interaction and Focal adhesion were found in 268 DC3. These collagen genes may serve as candidate biomarkers for telogen-anagen 269 regeneration. For example, a kind of self-assembling peptide hydrogel scaffold was 270 used to build the ECM environment in vitro to promote HF regeneration [61].

271 Pathway crosstalk through mRNA-IncRNA co-expression network in 272 cashmere growth cycle

273 A total of 16 signaling pathways, 145 pathway genes, and 93 co-expressed lncRNAs

274 (Pearson correlation $\geq 0.8$ ) (Additional file 7: Table S7) are enrolled in this pathway275 mRNA-lncRNA network (Figure 3). It can be seen that the DC1 sub-network has no 276 positive correlation with DC2 and DC3, which can be explained by the possible 277 antagonistic relationship between DC1 and DC2. The telogen-anagen transition phase 278 of DC3 overlaps with the telogen stage, so the DC3 subnet is closely connected to DC2.

279 Through this network, the function of lncRNAs can be inferred by their co-expressed 280 coding genes. Three novel lncRNAs (LNC.6206, LNC.8064 and LNC.16941) are 281 highly connected (by more than 6 coding genes) in the DC1 sub-network, indicating 282 that they may mediate in promoting HF development. Similarly, four novel lncRNAs 
(LOC106503915 and LOC108637283) were found with high degrees in the DC2 subnetwork. The coding genes connected to them cover all the signaling pathways in DC2, indicating that these lncRNAs are likely to be involved in the SHF degeneration. The PI3K-Akt and NF-kappa B signaling are both functioning in DC2 and DC3 subnetworks. 9 novel lncRNAs and 4 annotated lncRNAs connecting DC2 and DC3 may play a role in the transition from telogen to early anagen.

Expression differences triggered by melatonin reveal a potential signal pathway map regulating cashmere growth

292 A total of 908 genes were detected to have different expression patterns between the D 293 group and the M group (Figure 4A) (Additional file 8: Table S8). When treated with 294 melatonin, $80(24.6 \%)$ genes in DC3 maintained the same pattern as group D, but the expression patterns of most genes in DC1 and DC2 have changed (Figure 4B). Among 296 the 908 differential changing genes (DCGs), 369 genes belonging to the three clusters 297 DC1, DC2 and DC3 were divided into three clusters MC1 (159), MC2 (144) and MC3 298 (66), respectively (Figure 4C) (Additional file 9: Table S9). After melatonin treatment, $299 \mathrm{MC1}$ and MC2 in the M group lost the periodic expression fluctuations as in the D 300 group (Figure 4D). In Apr-May, gene expression of MC1 dropped to the lowest while 301 expression of MC2 rose to the highest in the D group and shedding appeared. Pathway 302 enrichment showed that MC1 and MC2 were involved in the promotion and regression 303 of HFs, respectively (Figure 4E) (Additional file 10: Table S10). In the M group, the 304 expression of MC1 was still rising and MC2 was falling from January to April, which 
may lead to a non-resting period after shedding in the $\mathrm{M}$ group. Therefore, genes in MC3, which may be responsible for restarting HF growth, did not show a significant

307 increase in expression in February-May. In September-November, the expression of $308 \mathrm{MC} 1$ in the M group was relatively lower than that in the D group, while the expression 309 of MC2 in the M group was higher than that in the D group, which may disrupt the 310 growth and maintenance of HFs and cause a local shedding.

311 To reveal the differential expression pattern every month, we use DESeq2 to obtain 312 monthly differentially expressed genes (monthly DEGs) between melatonin and control 313 groups with adjusted $p$-value (padj) $\leq 0.05$ and $\mid \log 2$ FoldChange $\mid \geqslant 1$. A total of 941 314 monthly DEGs were identified from monthly pairwise comparisons. The monthly

315 DEGs are mainly distributed from April and September (Figure 5A), which exactly 316 cover the whole anagen stage, and $96 \%$ of them are protein-coding genes. The KEGG 317 analysis results (Figure 5B) (Additional file 11: Table S11) showed that Hedgehog 318 related genes (SHH, PTCH2, PTCH1) and Wnt related genes (FZD10, WIF1, LEF1, 319 WNT11) were up-regulated in April, while the GO results (Figure 5C) (Additional 320 file 12: Table S12) showed that Hedgehog genes (PTCH2, FOXE1) and other related 321 up-regulated genes like FOXN1, HOXC13, KRT25 and KRT71 were significantly 322 enriched from April to May. Related studies have shown that Wnt [62-64] and Shh [46, 323 65] signaling can promote hair follicle cell division and the initiation of anagen; $\mathrm{C}$.

324 Potter et al. demonstrated that nude mutant gene FOXN1 is a regulatory target of 325 HOXC13 and the knockdown of both genes could cause abnormal hair growth [66]. Yu 326 et al. showed that a missense mutation at the helix terminus of KRT25 can cause a 
327 reduction of woolly hair [67]. KRT71 is an inner root sheath keratin, and the mutant of

328 KRT71 can disrupt keratin intermediate filament formation [68]. Therefore, the

329 Hedgehog related genes (SHH, PTCH2, PTCH1, FOXE1), Wnt related genes (FZD10,

330 WIF1, LEF1, WNT11), and other hair development related genes FOXN1, HOXC13,

331 KRT25 and KRT71 may be responsible for the initiation of a fast anagen progressing

332 stage from April to July. Meanwhile, the KEGG results (Figure 5B) showed that the

333 expression of ECM receptor interaction genes (COL6A3, THBS3, COL1A1, FRAS1,

334 FREM1/2) was downregulated from June to July. ECM is an important matrix required

335 for hair follicle cell growth. Xu et al. showed that the amount of ECM occupied by each

336 cell determined the volume size of dermal papilla (DP) in hair follicles [69], and Zhu

337 et al. demonstrated that the rapid growth of anagen hair follicles in cashmere goat

338 required high expression of ECM and cell surface proteins [1]. The down-regulation of

339 ECM-related genes from June to July may contribute to the inhibition of hair follicle

340 growth, and the down-regulation of genes involved in the Hedgehog (SHH, PTCH2)

341 and Wnt (WNT6, NOTUM, SFRP2) pathways indicated that hair follicle growth may

342 be inhibited in August. The expression of genes in chemokine signaling pathway

343 (CCL17, CCL22, CCL2, LYN, RAC2, LOC102170772, PIK3CG, VAV1) was up-

344 regulated in September. Experiments by Nagao et al. have shown that chemokines can

345 induce immune cell migration as the hair follicle enters the catagen phase [51], which

346 may further promote the hair follicle apoptosis. Therefore, the downregulated ECM

347 receptor interaction genes (COL6A3, THBS3, COL1A1, FRAS1, FREM1, FREM2),

348 Hedgehog genes (SHH, PTCH2), Wnt genes (WNT6, NOTUM, SFRP2) and up- 
regulated chemokines (CCL17, CCL22, CCL2, LYN, RAC2, LOC102170772,

350 PIK3CG, VAV1) may contribute to the second cashmere shedding happened from

351 August to November. Jin et al.'s transcriptome analysis of the melatonin-treated group

352 with MTC knockdown experiments confirmed that melatonin can promote hair follicle

353 development by activating the NF-kappa B pathway through promoting the expression

354 of MTC [70]. Genes in NF-kappa B signaling pathway (CD40LG, LTB,

355 LOC102176695, LYN, BTK) were up-regulated in September, which may contribute

356 to the hair follicle development after the second cashmere shedding. The representative

357 GO \& KEGG enrichment results of monthly DEGs mentioned above were summarized

358 in Table 1.

\section{Discussion}

360 In this study, the cashmere goat skin samples of the experimental group and the control

361 group covered 12 months, that is, the entire cashmere growth cycle. Many studies have

362 used RNA-seq to explore the differences in gene expression in different growth stages

363 of cashmere. For example, Geng et al. conducted a functional analysis of the differences

364 in gene expression between three developmental stages of hair follicles in cashmere

365 goats, and identified key genes that are involved in the regulation of cashmere growth

366 [71]. Zhang et al. performed transcriptome sequencing analysis on hair follicles in four

367 seasons and explored the regulation of seasonal variation genes on the cashmere growth

368 cycle of the cashmere goat and milk goat [72]. However, these studies only selected

369 three or more stages determined by experiments or experience at the cellular level.

370 There is still a lack of research on the in-depth exploration of the dynamic pattern of 
371 gene expression during different cashmere growth stages on the scale of whole cycles.

372 Therefore, this study performed transcriptome sequencing on the skin samples covering

373 the entire cashmere cycles for 12 months, which aims to explore the dynamics of gene

374 expression in the cashmere growth cycle in more detail.

375 The gene expression pattern for 12 months can provide useful information for 376 distinguishing different cashmere growth stages from the genetic and molecular levels.

377 According to the cluster-month correlations in Figure 2C, we grouped the cashmere 378 growth cycle into three main stages: (1) anagen (April-October); (2) catagen and 379 telogen (October-December and January-April); (3) telogen-anagen regeneration 380 (February-May). The corresponding gene clusters are DC1, DC2 and DC3, respectively.

381 Some canonical pathways such as the Wnt, TGF-beta and Hippo signaling pathways 382 are enriched in DC1. DC2 genes are mainly enriched in pathways such as Cell adhesion 383 molecules (CAMs), Cytokine-cytokine receptor interaction, Jak-STAT, Fc epsilon RI, 384 NOD-like receptor, Rap1, PI3K-Akt, cAMP, NF-kappa B and many immune-related 385 pathways. Interestingly, due to the overlap of the early anagen and telogen-anagen 386 regeneration stages, the genes of DC3 and DC2 were partially enriched in several same 387 pathways like CAMs, Focal adhesion, extracellular matrix (ECM)-receptor interaction, 388 PI3K-Akt and NF-kappa B signaling. Besides, by constructing a co-expression network 389 of genes (that are enriched in key pathways) and lncRNAs in three clusters, we reveal 390 the possible regulators for crosstalk between different signaling pathways, and 391 unearthed novel lncRNAs that may participate in these pathways.

392 In addition to unraveling the gene expression regulation mechanisms of the transition 
393 between different stages of the hair follicle cycle, this study also helps to figure out the 394 role of exogenous melatonin in the specific stages of the cashmere growth cycle. By 395 identifying genes that exhibit different expression patterns during the cashmere growth 396 cycle under the stimulation of melatonin, we also obtained three gene clusters (MC1, 397 MC2 and MC3) that may affect the cashmere growth cycle. Among them, MC1 genes 398 (BAMBI, BMP2, BMP8A, FZD10, LEF1, PPP2R1B, SMAD6 and WNT11) and MC2 399 genes (IL6R, IL7R, IL11RA, IL15, IL18, PDE1A, PDE1B and PDE3B) showed 400 opposite periodicity in group D. However, after the melatonin treatment, this regular 401 fluctuation has been disordered. MC3 genes (COL1A1, COL1A2, COL3A1, CHAD, 402 CREB3L1 and THBS3) were expressed specifically in the anagen restart phase (Apr403 May) in group D, but there was no similarly significant expression pattern in group M. 404 The relative expression levels of monthly DEGs (Additional file 13) show that the hair 405 development related genes HOXC13, KRT25, KRT71, FOXN1 were generally 406 expressed at higher levels at the beginning of fast anagen progressing period from April 407 to May, implying that they may function to promote the initiation of anagen. Wnt genes 408 (Wif-1, WNT11, FZD10, LEF1, NOTUM, SFRP2, WNT6) together with Hedgehog 409 genes (SHH, PTCH1, PTCH2, FOXE1) showed higher expression levels between April 410 and May, but decreased in August, which implied that Wnt-related genes may promote 411 the rapid transition into anagen phase of hair follicles between April and May, and 412 repress the growth of hair follicles on the eve of the second cashmere shedding period 413 in August. Chemokines (CCL17, CCL22, CCL2, LYN, RAC2, LOC102170772, 414 PIK3CG and VAV1) and NF-kB genes (ZAP70, LYN, BTK, CD40LG, LTB) were 
415 highly expressed in September. The NF- $\kappa B$ pathway may facilitate the progress of the 416 subsequent cashmere growth phase. Meanwhile, chemokines such as LTN may 417 promote the second cashmere shedding.

418 KEGG pathway could be used as a reference to demonstrate the regulatory relationships 419 of differentially expressed genes. Taking the above results together and collating the 420 relevant KEGG pathway visualization results (Additional file 14), here we proposed a 421 signaling pathway diagram of melatonin influenced cashmere growth cycle (Figure 6), 422 which covered the main differentially expressed genes related to cashmere growth in 423 anagen phase from April to September. The anagen phase of melatonin-treated groups 424 was composed of a fast anagen progressing stage and a second cashmere shedding stage. 425 The fast anagen progressing stage was from April to July, and this period was 426 characterized by the occurrence of the first massive cashmere shedding at the end of 427 April, and the rapid transition into anagen phase of hair follicles from May to July, 428 when the quick resumption of cashmere growth appeared instead of residing in a resting 429 non-growth period. The rapid resumption of the anagen phase of hair follicles may be 430 due to the high expression of KRT25, HOXC13 and HOXC13's regulatory target 431 FOXN1, high expression of FZD10, WIF1, LEF1, WNT11 in Wnt signaling pathway, 432 and SHH, PTCH1, PTCH2, FOXE1 in sonic hedgehog signaling pathway. The second 433 cashmere shedding period was from August to September. The appearance of the 434 second cashmere shedding may not only be associated with the low expression of ECM 435 signaling molecules such as FREM1, FREM2, FRAS1, COL1A1, COL6A3, THBS3 in 436 June and July, sonic hedgehog signaling pathway genes such as SHH, PTCH2 and 
WNT signaling pathway genes such as NOTUM, SFRP2, WNT6 in August, but also with the high expression of chemokines such as CCL, LYN, PIK3CG, VAV1, RAC2 in August. In addition, the highly expressed genes in NF-kappa B signaling pathway such as CD40LG, LTB, ZAP, LYN, BTK in September may promote the subsequent growth of cashmere after the second cashmere shedding period.

\section{Conclusions}

In summary, this study systematically analyzed RNA-seq data from skin samples of cashmere goats covering the entire cashmere growth cycle and identified a series of key regulators (including genes and lncRNAs) that may be involved in the cashmere growth processes. Based on gene expression patterns, we elucidated a more precise division of the cashmere growth cycle from the molecular level. However, due to the lack of sampling points, some key stages (especially the transition state) are still not well identified. A possible way is to increase the sampling time density before and after the stage of interest. In addition, differences in individual development, such as different growth rates, may also cause bias in the monthly differential expression analysis.

\section{Abbreviations}

SCG: Seasonal changing gene; CAM: Cell adhesion molecule; DEG: Differentially expressed gene; Monthly DEG: Monthly differentially expressed gene; ECM: Extracellular matrix; HF: Hair follicle; SHF: Secondary hair follicle; BMP: Bone morphogenetic protein; SHH: Sonic hedgehog; FGF: Fibroblast growth factor; TGF: Transforming growth factor; KRT: Keratin; KRTAP: Keratin-associated protein; lncRNA: Long noncoding RNA; miRNA: MicroRNA; WGCNA: Weighted gene co- 
459

460 Gene Ontology; BP: Biological process; PCA: Principal components analysis; LEF:

461 Lymphoid enhancer binding factor; IL: Interleukin; DKK: Dickkopf

\section{Declarations}

\section{Ethics approval and consent to participate}

464 The skin samples in this study were collected from the Inner Mongolia Cashmere Goats.

465 All procedures were conducted according to the standards of the Animal Care and Use

466 Committee in Inner Mongolia University for Nationalities, China.

\section{Consent for publication}

468 Not applicable.

469 Availability of data and materials

470 The authors acknowledge that the data presented in this study must be deposited and 471 made publicly available in an acceptable repository, prior to publication.

\section{Competing interests}

473 The authors declare that they have no competing interests.

\section{$474 \quad$ Funding}

475 This work has been funded under the National Natural Science Foundation of China 476 (\#31402052), and the Natural Science Foundation of Inner Mongolia (\#2019MS03078, 477 \#2019BS03006, \#2019LH03012), and the Special Open Project on the Construction of 478 Mongolian Pharmacy Doctoral Station for National Service with Special Needs 479 (\#MDMYBJ2019004), and the Doctoral Scientific Research Foundation of Inner 480 Mongolia University for Nationalities (BS308) provided support for the design of the 
481 study and data collection.

482 Authors' contributions

483 Chun Li conducted the research, Cong Feng and Guangyuan Ma analysed the data and 484 prepare the manuscript, Shaoyin Fu helped RNA-seq and data processing, Jinquan Li 485 and Wenguang Zhang provided the test platform, Ming Chen supervised data analysis 486 and manuscript revising.

487 Acknowledgments

488 The authors are thankful for the samples provided by the Hanshan White Cashmere 489 Goat Breeding Farm.

$490 \quad$ Author details

$491{ }^{1}$ College of Animal Science and Technology, Inner Mongolia University for 492 Nationalities, Tongliao 028000, China. ${ }^{2}$ College of Life Sciences, Zhejiang University, 493 Hangzhou 310058, China. ${ }^{3}$ Inner Mongolia Academy of Agricultural \& Animal 494 Husbandry Sciences, Hohhot 010018, China. ${ }^{4}$ College of Life Science and Food 495 Engineering, Inner Mongolia University for Nationalities, Tongliao 028000, China. $496{ }^{5}$ College of Animal Science, Inner Mongolia Agricultural University, Hohhot 010018, 497 China. 


\section{References}

504 1. Zhu B, Xu T, Yuan J, Guo X, Liu D. Transcriptome Sequencing Reveals 505 Differences between Primary and Secondary Hair Follicle-derived Dermal Papilla Cells 506 of the Cashmere Goat (Capra hircus). PLoS One. 2013;8(9):e76282.

507 2. Bykov YS, Schaffer M, Dodonova SO, Albert S, Plitzko JM, Baumeister W, et al. 508 The structure of the COPI coat determined within the cell. eLife. 2017;6.

509 3. Pin D, Cachon T, Carozzo C. Determination of the Depth of Excision Using a 510 Dermatome (Aesculap $\left.{ }^{\circledR}\right)$ to Export all Hair Follicle Bulbs from a Donor Site in the Dog. 511 J Vet Med A. 2007;54(9):539-41.

512 4. McCarthy B. Specialty animal fibers. Textiles. 1998;20(1):6-8.

513 5. Yang F, Liu Z, Zhao M, Mu Q, Che T, Xie Y, et al. Skin transcriptome reveals the 514 periodic changes in genes underlying cashmere (ground hair) follicle transition in 515 cashmere goats. BMC Genomics. 2020;21(1).

516 6. Klören WRL, Norton BW. Melatonin and fleece growth in Australian cashmere 517 goats. Small Rumin Res. 1995;17(2):179-85.

518 7. Betteridge K, Welch R, Pomroy W, Lapwood K, Devantier B, editors. Out of 519 season cashmere growth in feral goats. Proceedings of the second international 520 cashmere conference; 1987.

521 8. Litherland AJ, Paterson DJ, Parry AL, Dick HB, Staples LD. Melatonin for 522 cashmere production. Proceedings of the New Zealand Society of Animal Production. $523 \quad 1990 ; 50: 339-43$.

524 9. Welch RAS, Gurnsey MP, Betteridge K, Mitchell RJ. Goat fibre response to 
melatonin given in spring in two consecutive years. Proceedings of the New Zealand

526 Society of Animal Production. 1990;50:335-8.

527 10. Yang CH, Duan CH, Wu ZY, Li Y, Luan YY, Fu XJ, et al. Effects of melatonin 528 administration to cashmere goats on cashmere production and hair follicle 529 characteristics in two consecutive cashmere growth cycles. Domest Anim Endocrinol. $530 \quad 2021 ; 74: 106534$.

531 11. Duan $\mathrm{CH}, \mathrm{Xu}$ JH, Sun CM, Jia ZH, Zhang W. Effects of melatonin implantation 532 on cashmere yield, fibre characteristics, duration of cashmere growth as well as growth 533 and reproductive performance of Inner Mongolian cashmere goats. J Anim Sci 534 Biotechnol. 2015;6.

535 12. Tsai S-Y, Sennett R, Rezza A, Clavel C, Grisanti L, Zemla R, et al. Wnt/ $\beta$-catenin 536 signaling in dermal condensates is required for hair follicle formation. Dev Biol. $537 \quad 2014 ; 385(2): 179-88$.

538 13. Enshell-Seijffers D, Lindon C, Kashiwagi M, Morgan BA. $\beta$-catenin Activity in 539 the Dermal Papilla Regulates Morphogenesis and Regeneration of Hair. Dev Cell. $540 \quad 2010 ; 18(4): 633-42$.

541 14. Veltri A, Lang C, Lien W-H. Concise Review: Wnt Signaling Pathways in Skin 542 Development and Epidermal Stem Cells. Stem Cells. 2018;36(1):22-35.

543 15. Yuhki M. BMPR1A signaling is necessary for hair follicle cycling and hair shaft 544 differentiation in mice. Development. 2004;131(8):1825-33.

545 16. Kulessa H. Inhibition of Bmp signaling affects growth and differentiation in the 546 anagen hair follicle. EMBO J. 2000;19(24):6664-74. 
547 17. Woo WM, Zhen HH, Oro AE. Shh maintains dermal papilla identity and hair 548 morphogenesis via a Noggin-Shh regulatory loop. Genes Dev. 2012;26(11):1235-46.

549 18. Żak M, Klis SFL, Grolman W. The Wnt and Notch signalling pathways in the 550 developing cochlea: Formation of hair cells and induction of regenerative potential. Int 551 J Dev Neurosci. 2015;47(Part_B):247-58.

552 19. Rosenquist TA, Martin GR. Fibroblast growth factor signalling in the hair growth 553 cycle: Expression of the fibroblast growth factor receptor and ligand genes in the 554 murine hair follicle. Dev Dyn. 1996;205(4):379-86.

555 20. Choi YM, Choi SY, Kim H, Kim J, Ki MS, An I-S, et al. TGF $\beta$ family mimetic 556 peptide promotes proliferation of human hair follicle dermal papilla cells and hair 557 growth in C57BL/6 mice. Biomedical Dermatology. 2018;2(1).

558 21. Wang J, Sui J, Mao C, Li X, Chen X, Liang C, et al. Identification of Key Pathways 559 and Genes Related to the Development of Hair Follicle Cycle in Cashmere Goats. 560 Genes. 2021;12(2):180.

561 22. Nie Y, Li S, Zheng X, Chen W, Li X, Liu Z, et al. Transcriptome Reveals Long 562 Non-coding RNAs and mRNAs Involved in Primary Wool Follicle Induction in Carpet 563 Sheep Fetal Skin. Front Physiol. 2018;9.

564 23. Yin R, Wang Y, Zhao S, Yin R, Bai M, Wang Z, et al. LncRNA-599554 sponges 565 miR-15a-5p to contribute inductive ability of dermal papilla cells through positively 566 regulating the expression of Wnt3a in cashmere goat. Electron J Biotechnol. 2020;45. 567 24. Wang S, Ge W, Luo Z, Guo Y, Jiao B, Qu L, et al. Integrated analysis of coding 568 genes and non-coding RNAs during hair follicle cycle of cashmere goat (Capra hircus). 
BMC Genomics. 2017;18(1).

25. Wang J, Che L, Hickford J, Zhou H, Hao Z, Luo Y, et al. Identification of the Caprine Keratin-Associated Protein 20-2 (KAP20-2) Gene and Its Effect on Cashmere Traits. Genes. 2017;8(11):328.

26. Sulayman A, Tian K, Huang X, Tian Y, Xu X, Fu X, et al. Genome-wide identification and characterization of long non-coding RNAs expressed during sheep fetal and postnatal hair follicle development. Sci Rep. 2019;9(1).

27. Andrews S. FastQC: a quality control tool for high throughput sequence data. 2010.

28. Pertea M, Kim D, Pertea GM, Leek JT, Salzberg SL. Transcript-level expression analysis of RNA-seq experiments with HISAT, StringTie and Ballgown. Nat Protoc. 2016;11(9):1650.

29. Kang Y-J, Yang D-C, Kong L, Hou M, Meng Y-Q, Wei L, et al. CPC2: a fast and accurate coding potential calculator based on sequence intrinsic features. Nucleic Acids Res. 2017;45(W1):W12-W6.

30. Sun L, Luo H, Bu D, Zhao G, Yu K, Zhang C, et al. Utilizing sequence intrinsic composition to classify protein-coding and long non-coding transcripts. Nucleic Acids Res. 2013;41(17):e166.

31. Anders S, Pyl PT, Huber W. HTSeq — a Python framework to work with highthroughput sequencing data. Bioinformatics. 2015;31(2):166-9.

32. Love MI, Huber W, Anders S. Moderated estimation of fold change and dispersion for RNA-seq data with DESeq2. Genome Biol. 2014;15(12):1-21. 
591 analysis. BMC Bioinformatics. 2008;9(1):1-13.

592 34. Huang DW, Sherman BT, Tan Q, Kir J, Liu D, Bryant D, et al. DAVID

593 Bioinformatics Resources: expanded annotation database and novel algorithms to better 594 extract biology from large gene lists. Nucleic Acids Res. 2007;35(Web Server 595 issue):W169-W75.

596 35. Kanehisa M, Goto S. KEGG: kyoto encyclopedia of genes and genomes. Nucleic 597 Acids Res. 2000;28(1):27-30.

598 36. Ashburner M, Ball CA, Blake JA, Botstein D, Butler H, Cherry JM, et al. Gene 599 Ontology: Tool for the unification of biology. Nat Genet. 2000;25(1):25-9.

600 37. Yu G, Wang L-G, Han Y, He Q-Y. ClusterProfiler: an R Package for Comparing 601 Biological Themes Among Gene Clusters. OMICS: J Integrative Biol. 2012;16(5):2846027.

603 38. Morgan M, Shepherd L. AnnotationHub: Client to access AnnotationHub 604 resources. https://bioconductor.org/packages/AnnotationHub/2020 [

605 39. Shannon P, Markiel A, Ozier O, Baliga NS, Wang JT, Ramage D, et al. Cytoscape: 606 a software environment for integrated models of biomolecular interaction networks. 607 Genome Res. 2003;13(11):2498-504.

608 40. Oshimori N, Fuchs E. Paracrine TGF-beta signaling counterbalances BMP609 mediated repression in hair follicle stem cell activation. Cell Stem Cell. 2012;10(1):6361075.

611 41. Rishikaysh P, Dev K, Diaz D, Qureshi WM, Filip S, Mokry J. Signaling involved 612 in hair follicle morphogenesis and development. Int J Mol Sci. 2014;15(1):1647-70. 
613 42. Zhang Y, Yu J, Shi C, Huang Y, Wang Y, Yang T, et al. Lefl contributes to the 614 differentiation of bulge stem cells by nuclear translocation and cross-talk with the 615 Notch signaling pathway. Int J Med Sci. 2013;10(6):738-46.

616 43. Attisano L, Wrana JL. Signal integration in TGF-beta, WNT, and Hippo pathways. 617 F1000Prime Rep. 2013;5:17.

618 44. Hwang J, Mehrani T, Millar SE, Morasso MI. Dlx3 is a crucial regulator of hair 619 follicle differentiation and cycling. Development. 2008;135(18):3149-59.

620 45. Guo T, Han J, Yuan C, Liu J, Niu C, Lu Z, et al. Comparative proteomics reveals 621 genetic mechanisms underlying secondary hair follicle development in fine wool sheep 622 during the fetal stage. J Proteomics. 2020;223:103827.

623 46. St-Jacques B, Dassule H, Karavanova I, Botchkarev V, Li J, Danielian P, et al. 624 Sonic hedgehog signaling is essential for hair development. Curr Biol. 625 1998;8(19):1058-69.

626 47. Nanba D, Nakanishi Y, Hieda Y. Role of Sonic hedgehog signaling in epithelial 627 and mesenchymal development of hair follicles in an organ culture of embryonic mouse 628 skin. Dev Growth Differ. 2003;45(3):231-9.

629 48. Abe Y, Tanaka N. Roles of the Hedgehog Signaling Pathway in Epidermal and 630 Hair Follicle Development, Homeostasis, and Cancer. J Dev Biol. 2017;5(4).

631 49. Zhu B, Xu T, Zhang Z, Ta N, Gao X, Hui L, et al. Transcriptome sequencing 632 reveals differences between anagen and telogen secondary hair follicle-derived dermal 633 papilla cells of the Cashmere goat (Capra hircus). Physiol Genomics. 2014;46(3):10463411. 
50. Mueller SN, Germain RN. Stromal cell contributions to the homeostasis and

636 functionality of the immune system. Nat Rev Immunol. 2009;9(9):618-29.

637 51. Nagao K, Kobayashi T, Moro K, Ohyama M, Adachi T, Kitashima DY, et al.

638 Stress-induced production of chemokines by hair follicles regulates the trafficking of 639 dendritic cells in skin. Nat Immunol. 2012;13(8):744-52.

640 52. Scharschmidt TC, Vasquez KS, Pauli ML, Leitner EG, Chu K, Truong HA, et al.

641 Commensal Microbes and Hair Follicle Morphogenesis Coordinately Drive Treg 642 Migration into Neonatal Skin. Cell Host Microbe. 2017;21(4):467-77 e5.

643 53. Harel S, Higgins CA, Cerise JE, Dai Z, Chen JC, Clynes R, et al. Pharmacologic 644 inhibition of JAK-STAT signaling promotes hair growth. Sci Adv. 2015;1(9):e1500973. 645 54. Wang ECE, Dai Z, Ferrante AW, Drake CG, Christiano AM. A Subset of TREM2( $\left(^{+}\right)$ 646 Dermal Macrophages Secretes Oncostatin M to Maintain Hair Follicle Stem Cell 647 Quiescence and Inhibit Hair Growth. Cell Stem Cell. 2019;24(4):654-69 e6.

648 55. Kim JE, Lee YJ, Park HR, Lee DG, Jeong KH, Kang H. The Effect of JAK 649 Inhibitor on the Survival, Anagen Re-Entry, and Hair Follicle Immune Privilege 650 Restoration in Human Dermal Papilla Cells. Int J Mol Sci. 2020;21(14).

651 56. Kwack MH, Kim MK, Kim JC, Sung YK. Dickkopf 1 promotes regression of hair 652 follicles. J Invest Dermatol. 2012;132(6):1554-60.

653 57. Kwack MH, Lee JH, Seo CH, Kim JC, Kim MK, Sung YK. Dickkopf-1 is involved 654 in dexamethasone-mediated hair follicle regression. Exp Dermatol. 2017;26(10):952-4. 655 58. Grosbois J, Demeestere I. Dynamics of PI3K and Hippo signaling pathways during 656 in vitro human follicle activation. Hum Reprod. 2018;33(9):1705-14. 
657

658

659

660

661

662

663

664

665

666

667

668

669

670

671

672

673

674

675

676

677

678

59. Chen Y, Fan Z, Wang X, Mo M, Zeng SB, Xu RH, et al. PI3K/Akt signaling pathway is essential for de novo hair follicle regeneration. Stem Cell Res Ther. 2020;11(1):144.

60. Nelson AM, Reddy SK, Ratliff TS, Hossain MZ, Katseff AS, Zhu AS, et al. dsRNA Released by Tissue Damage Activates TLR3 to Drive Skin Regeneration. Cell Stem Cell. 2015;17(2):139-51.

61. Wang X, Wang J, Guo L, Wang X, Chen H, Wang X, et al. Self-assembling peptide hydrogel scaffolds support stem cell-based hair follicle regeneration. Nanomedicine. 2016;12(7):2115-25.

62. Millar SE, Willert K, Salinas PC, Roelink H, Nusse R, Sussman DJ, et al. WNT Signaling in the Control of Hair Growth and Structure. Dev Biol. 1999;207(1):133-49. 63. Stenn KS, Paus R. Controls of Hair Follicle Cycling. Physiol Rev. 2001;81(1):44994.

64. Li Y-H, Zhang K, Yang K, Ye J-X, Xing Y-Z, Guo H-Y, et al. AdenovirusMediated Wnt10b Overexpression Induces Hair Follicle Regeneration. J Invest Dermatol. 2013;133(1):42-8.

65. Mill P. Sonic hedgehog-dependent activation of Gli2 is essential for embryonic hair follicle development. Genes Dev. 2003;17(2):282-94.

66. Potter CS, Pruett ND, Kern MJ, Baybo MA, Godwin AR, Potter KA, et al. The Nude Mutant Gene Foxn1 Is a HOXC13 Regulatory Target during Hair Follicle and Nail Differentiation. J Invest Dermatol. 2011;131(4):828-37. 
679 the Helix Termination Motif of KRT25 Causes Autosomal Dominant Woolly 680 Hair/Hypotrichosis. J Invest Dermatol. 2018;138(1):230-3.

681 68. Fujimoto A, Farooq M, Fujikawa H, Inoue A, Ohyama M, Ehama R, et al. A 682 Missense Mutation within the Helix Initiation Motif of the Keratin K71 Gene Underlies 683 Autosomal Dominant Woolly Hair/Hypotrichosis. J Invest Dermatol. $684 \quad 2012 ; 132(10): 2342-9$.

685 69. Xu T, Guo X, Wang H, Hao F, Du X, Gao X, et al. Differential gene expression 686 analysis between anagen and telogen of Capra hircus skin based on the de novo 687 assembled transcriptome sequence. Gene. 2013;520(1):30-8.

688 70. Jin M, Cao M, Cao Q, Piao J, Zhao F, Piao Ja. Long noncoding RNA and gene 689 expression analysis of melatonin-exposed Liaoning cashmere goat fibroblasts 690 indicating cashmere growth. The Science of Nature. 2018;105(9):60.

691 71. Geng RQ, Yuan C, Chen YL. Exploring Differentially Expressed Genes by RNA692 Seq in Cashmere Goat (Capra hircus) Skin during Hair Follicle Development and 693 Cycling. PLoS One. 2013;8(4).

694 72. Su R, Fan YX, Qiao X, Li XK, Zhang L, Li C, et al. Transcriptomic analysis reveals 695 critical genes for the hair follicle of Inner Mongolia cashmere goat from catagen to 696 telogen. PLoS One. 2018;13(10). 
701

702

703

704

705

706

707

708

709

710 711 November.

712

713

714

715

716

\section{Figures}

Figure 1. Overview of the transcriptome analysis. (A) The control and melatonin groups and sampling time. (B) Seasonal changing genes (SCGs) in the D and M groups.

(C) The number of coding genes, novel lncRNAs and annotated lncRNAs in the D and M groups. The Venn plot indicates that 759 genes are detected in both D and M groups. (D) PCA plot of the median-normalized samples in the D group. The circle suggests the direction of samples in cashmere growth stages. (E) PCA plot of the mediannormalized samples in the M group. (F) The cashmere length change plot in the D group. The shedding occurs in May-June. (G) The cashmere length change plot in the M group. The first shedding occurs in May-June. Another shedding appears in September-

Figure 2. Stage-specific gene expression patterns and pathway enrichment. (A)

Heatmap of the expression level of three D clusters (DC1, DC2 and DC3) in D and M groups. (B) The number of coding genes, annotated lncRNAs and novel lncRNAs in three D clusters. (C) KEGG pathway enrichment of the genes in DC1, DC2 and DC3. The pathways shared by DC2 and DC3 are shown in black. (D) The expression trends of three gene clusters. The cashmere growth cycle is divided into three stages according to the expression patterns.

Figure 3. Pathway-mRNA-IncRNA co-expression network. A total of 16 signaling pathways, 143 pathway genes, and 93 co-expressed lncRNAs (Pearson correlation $\geq 0.8$ ) 
723 are involved in this network. The size of the pathway node is positively related to its 724 degree.

Figure 4. Analysis of differences in the expression patterns triggered by melatonin.

727 (A) Venn diagrams showing SCGs in D clusters and M group and DCGs between D 728 and $\mathrm{M}$ groups. (B) The changed and unchanged genes of three D clusters after 729 melatonin treatment. (C) Heatmap of the expression level of three M clusters (MC1, 730 MC2 and MC3) in D and M groups. (D) The expression patterns of three M clusters in 731 D and M groups. (E) KEGG pathway enrichment of the genes in MC1, MC2 and MC3.

Figure 5. Detection of monthly differential expressed genes (DEGs) triggered by melatonin and pathway enrichment analysis. (A) The number and distribution of monthly differential expressed genes triggered by melatonin between $\mathrm{M}$ and $\mathrm{D}$ group.

(B) The KEGG pathway enrichment analysis of monthly differential expressed genes.

(C) The GO biological process enrichment analysis of monthly differential expressed genes. Note that up-regulated and down-regulated genes in different months are separated for GO \& KEGG analysis, in which 'Up' represents up-regulated genes, and

740 'Down' represents down-regulated genes. The color of nodes represents adjusted p-

741 value, and the size of nodes represents the ratio of gene numbers.

743 Figure 6. The putative pathway regulation model of the cashmere growth cycle

744 triggered by melatonin. The signal pathways related to cashmere shedding are marked 
745 with dotted line boxes, and the other solid line boxes mark the signal pathways which

746 may promote the hair follicle development.

748 Tables

749 Table 1. Summary of representative GO \& KEGG enrichment of monthly DEGs

\begin{tabular}{|c|c|c|c|}
\hline Cluster & Description & p.adjust & Gene Name \\
\hline Month4_Up & hair cycle & 0.0276 & $\begin{array}{l}\text { PTCH2, FOXE1, HOXC13, } \\
\text { FOXN1 }\end{array}$ \\
\hline Month5_Up & hair cycle & 0.0082 & $\begin{array}{l}\text { PTCH2, FOXE1, FOXN1, } \\
\text { HOXC13, KRT71, KRT25 }\end{array}$ \\
\hline Month4_Up & $\begin{array}{l}\text { Hedgehog signaling } \\
\text { pathway }\end{array}$ & 0.0306 & SHH, PTCH2, PTCH1 \\
\hline Month4_Up & Wnt signaling pathway & 0.0428 & $\begin{array}{l}\text { FZD10, WIF1, LEF1, } \\
\text { WNT11 }\end{array}$ \\
\hline Month6_Down & $\begin{array}{l}\text { ECM-receptor } \\
\text { interaction }\end{array}$ & 0.0259 & $\begin{array}{l}\text { COL6A3, THBS3, FREM1, } \\
\text { COL1A1 }\end{array}$ \\
\hline Month7_Down & $\begin{array}{l}\text { ECM-receptor } \\
\text { interaction }\end{array}$ & 0.0218 & FREM1, FRAS1, FREM2 \\
\hline Month8_Down & Wnt signaling pathway & 0.0257 & WNT6, NOTUM, SFRP2 \\
\hline Month8_Down & $\begin{array}{l}\text { Hedgehog signaling } \\
\text { pathway }\end{array}$ & 0.0318 & SHH, PTCH2 \\
\hline Month9_Up & $\begin{array}{l}\text { Chemokine } \\
\text { pathway }\end{array}$ & 0.0002 & $\begin{array}{l}\text { CCL17, CCL22, CCL2, LYN, } \\
\text { RAC2, LOC102170772, } \\
\text { PIK3CG, VAV1 }\end{array}$ \\
\hline Month9_Up & $\begin{array}{l}\mathrm{NF}-\kappa \mathrm{B} \\
\text { pathway }\end{array}$ & 0.0020 & $\begin{array}{l}\text { CD40LG, LTB, ZAP70, } \\
\text { LYN, BTK }\end{array}$ \\
\hline
\end{tabular}

750 Note: The first two lines are derived from GO BP results and others are derived from

751 KEGG results. P.adjust represents adjusted p-values. 


\section{Additional files}

757 Additional file 1: Table S1. Read counts of all detected genes in 72 samples.

758 Additional file 2: Table S2. Predicted lncRNAs.

759 Additional file 3: Table S3. Differentially expressed genes in the control group.

760 Additional file 4: Table S4. Differentially expressed genes in the melatonin-treated 761 group.

762 Additional file 5: Table S5. Detailed genes of 3 clusters in the control group (DC1, 763 DC2 and DC3).

764 Additional file 6: Table S6. The KEGG enrichment results of genes in DC1, DC2 and 765 DC3.

766 Additional file 7: Table S7. The Pearson correlations between genes and lncRNAs.

767 Additional file 8: Table S8. Genes with different expression patterns between the 768 control and melatonin-treated groups.

769 Additional file 9: Table S9. Detailed genes of 3 clusters in the melatonin-treated group $770 \quad(\mathrm{MC} 1, \mathrm{MC} 2$ and $\mathrm{MC} 3)$.

771 Additional file 10: Table S10. The KEGG enrichment results of genes in MC1, MC2 772 and MC3.

773 Additional file 11: Table S11. The KEGG analysis results of monthly DEGs.

774 Additional file 12: Table S12. The GO analysis results of monthly DEGs.

775 Additional file 13: Figure S1. Boxplots of relative expression levels of HOXC13(A), 776 KRT25(B), FOXN1(c), KRT71(D). Figure S2. Boxplots of relative expression levels 777 in WNT signaling pathway (Wif-1(A), WNT11(B), FZD10(C), LEF1(D), NOTUM(E), 
778 SFRP2(F), WNT6(G)). Figure S3. Boxplots of relative expression levels in Hedgehog

779 signaling pathway (Shh(A), PTCH1(B), PTCH2(C), FOXE1(D)). Figure S4. Boxplots

780 of relative expression levels of ECM related genes (FREM1(A), FREM2(B), 781 FRAS1(C), COL1A1(D), COL6A3(E), THBS3(F)). Figure S5. Boxplots of relative 782 expression levels of Chemokine related genes (CCL22(A), LYN(B), PIK3CG(C), 783 VAV1(D), RAC2(E), CCL17(F), CCL2(G), LOC102170772(H)). Figure S6. Boxplots

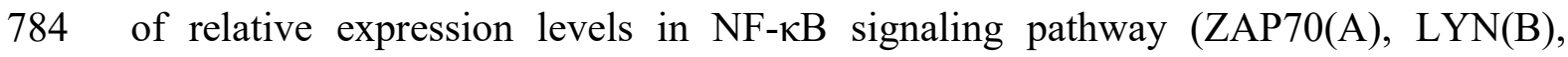
785 BTK(C), CD40LG(D), LTB(E)).

786 Additional file 14: Figure S1. Chemokine Signaling Pathway virtualized by KEGG 787 Pathway Maps. Figure S2. ECM-Receptor Interaction Signaling Pathway virtualized 788 by KEGG Pathway Maps. Figure S3. NF-Kappa B Signaling Pathway virtualized by 789 KEGG Pathway Maps. Figure S4. Rap1 Signaling Pathway virtualized by KEGG 790 Pathway Maps. Figure S5. Hedgehog Signaling Pathway virtualized by KEGG 791 Pathway Maps. Figure S6. Wnt Signaling Pathway virtualized by KEGG Pathway 792 Maps. High-expressed monthly DEGs are highlighted with pink color, while low793 expressed monthly DEGs are highlighted with blue color. 


\section{Figures}

A Control group (D)

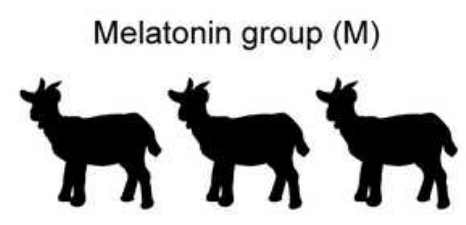

Monthly sampling

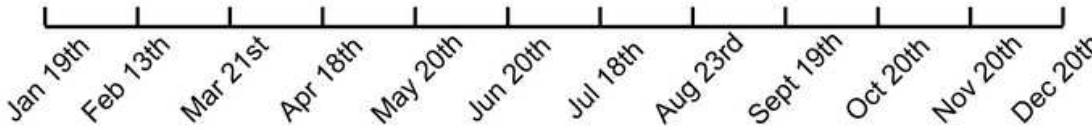

B

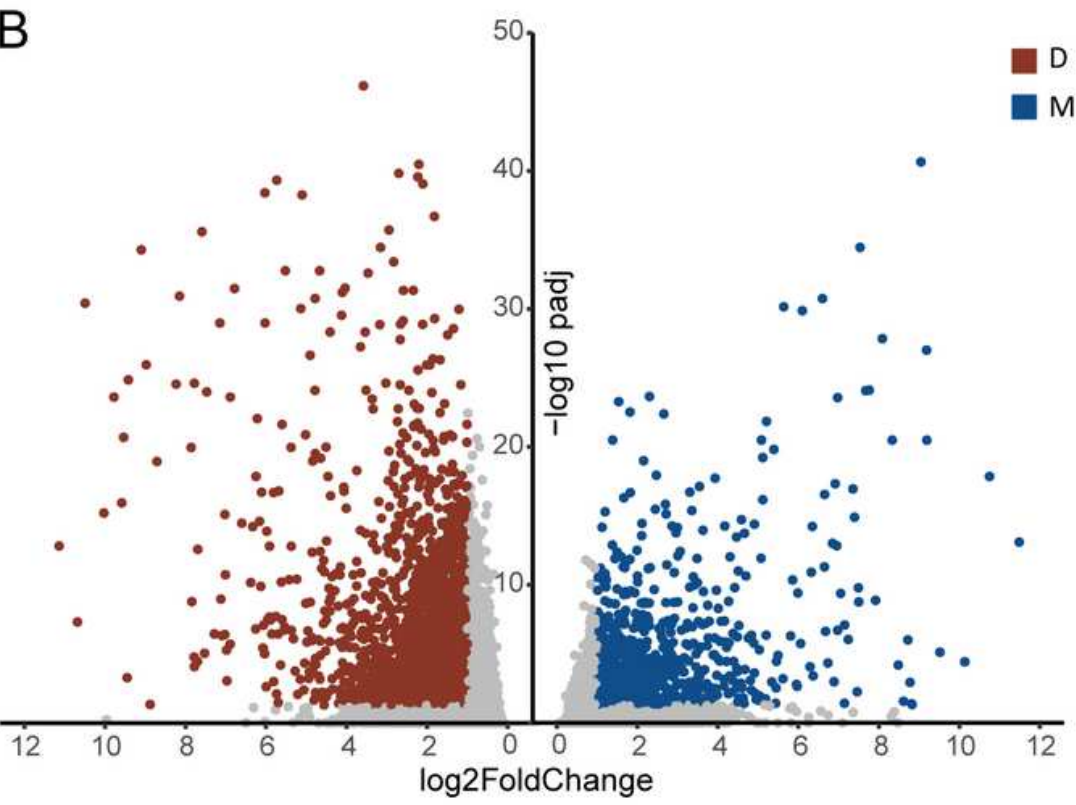

C

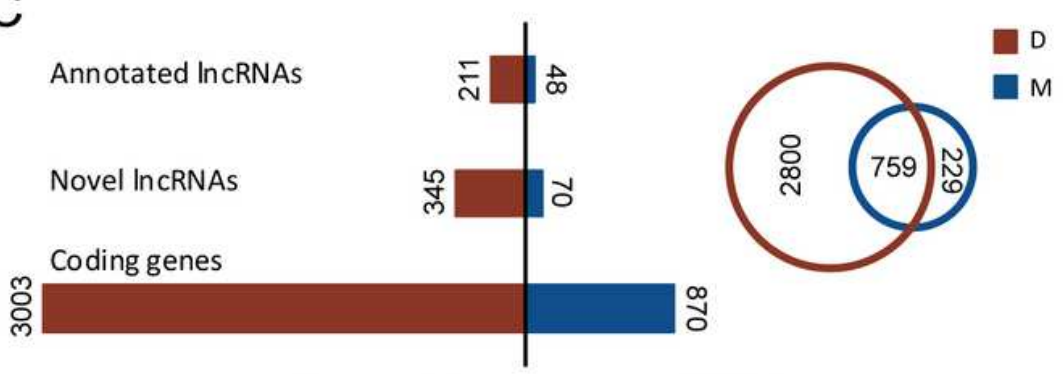

Seasonal changing genes (SCGs)

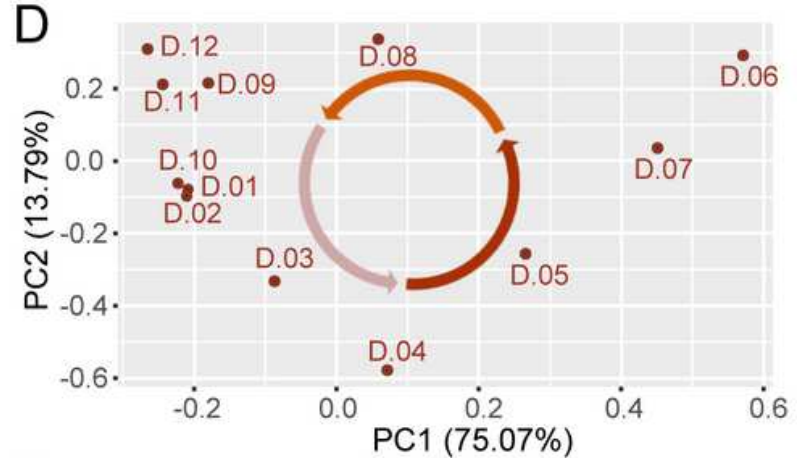

$\mathrm{E}$
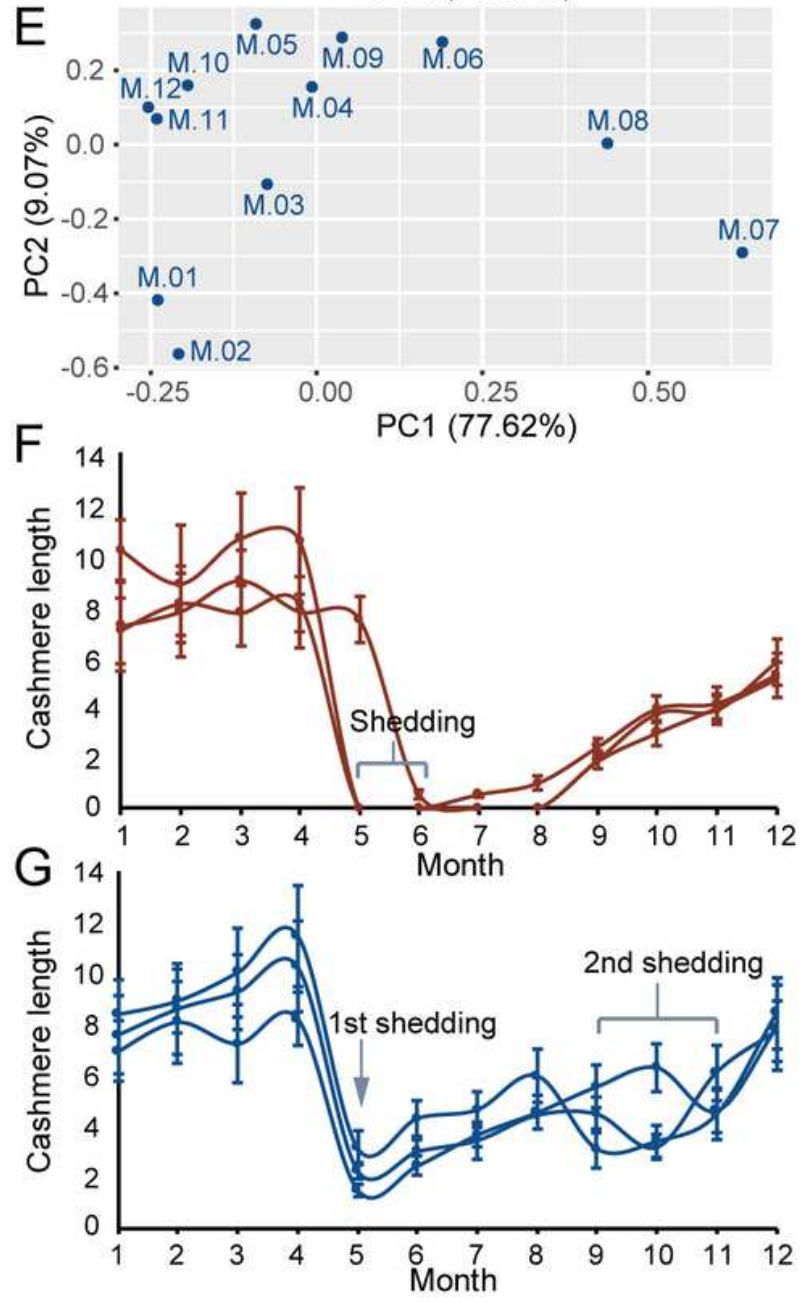

\section{Figure 1}

Overview of the transcriptome analysis. (A) The control and melatonin groups and sampling time. (B) Seasonal changing genes (SCGs) in the $D$ and $M$ groups. (C) The number of coding genes, novel IncRNAs and annotated IncRNAs in the D and M groups. The Venn plot indicates that 759 genes are detected in both $D$ and $M$ groups. (D) PCA plot of the median-normalized samples in the D group. The circle suggests the direction of samples in cashmere growth stages. (E) PCA plot of the median normalized samples in the $M$ group. (F) The cashmere length change plot in the $D$ group. The shedding occurs in May-June. (G) 
The cashmere length change plot in the M group. The first shedding occurs in May-June. Another shedding appears in September- November.

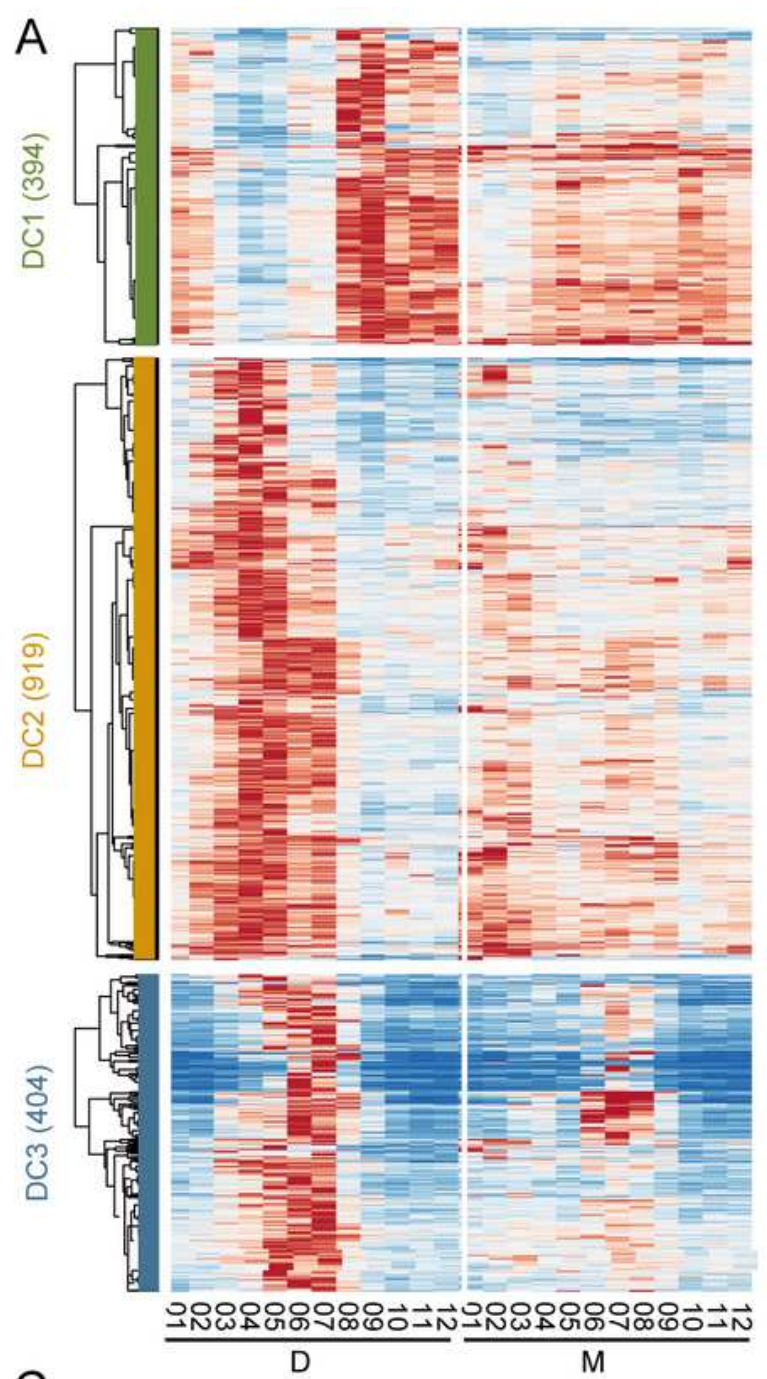

C

\section{DC1} $\mathrm{DC} 2$
$\mathrm{DC} 3$

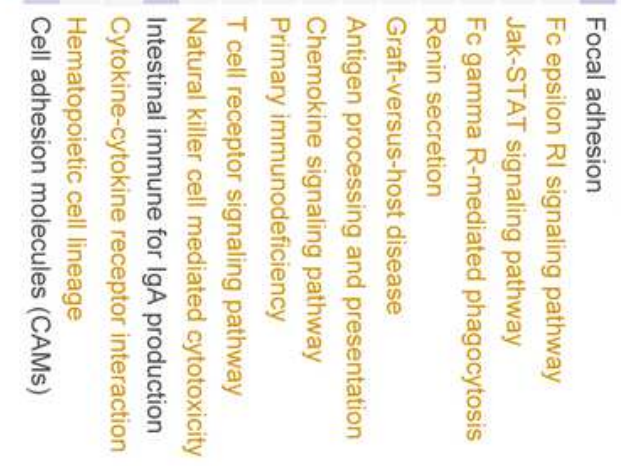

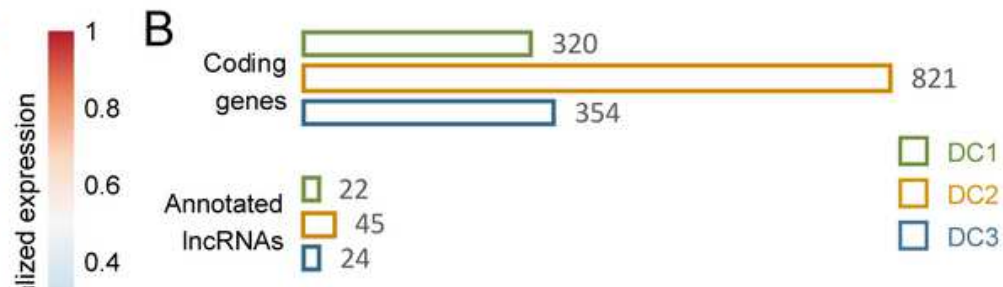
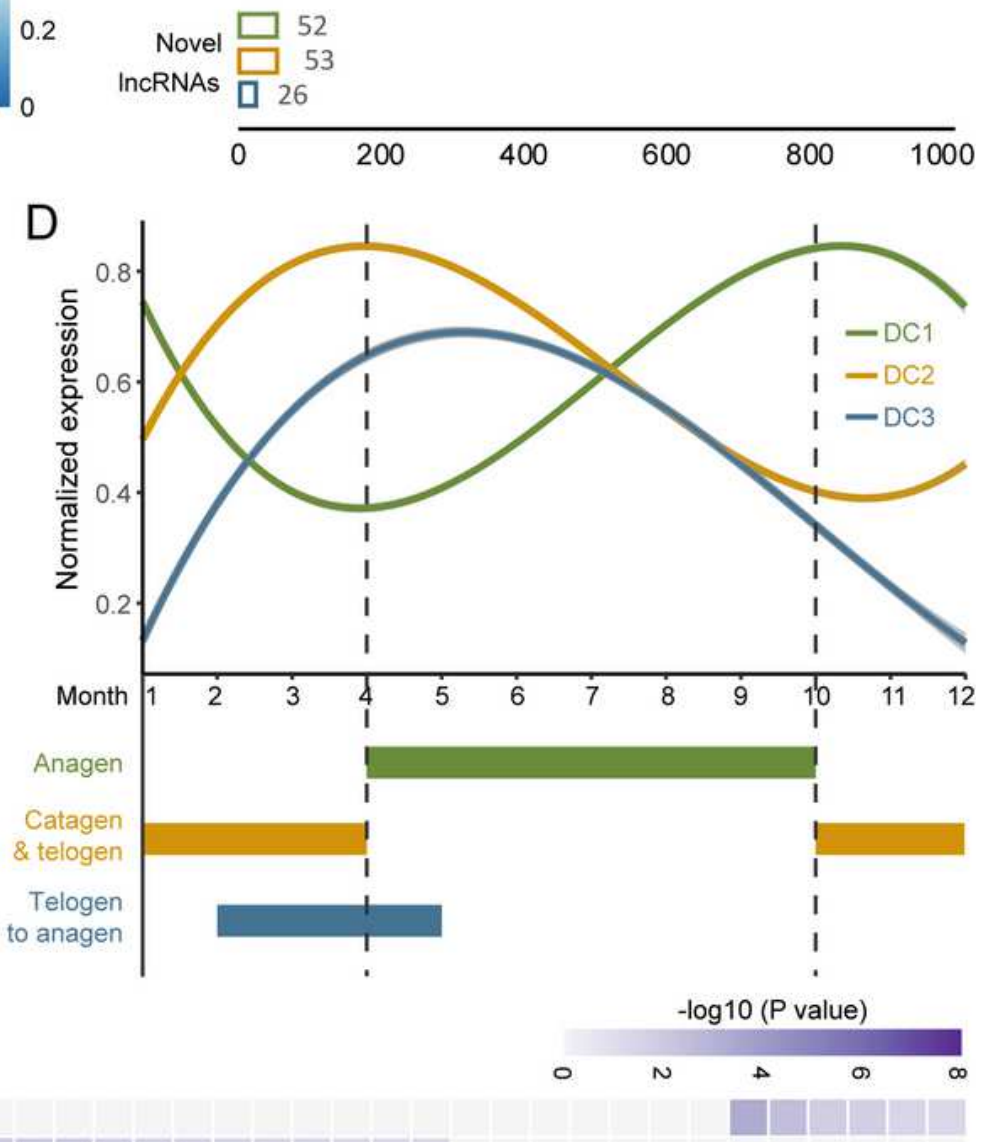

\section{Figure 2}

Stage-specific gene expression patterns and pathway enrichment. (A) Heatmap of the expression level of three D clusters (DC1, DC2 and DC3) in D and M groups. (B) The number of coding genes, annotated IncRNAs and novel IncRNAs in three D clusters. (C) KEGG pathway enrichment of the genes in DC1, DC2 
and DC3. The pathways shared by DC2 and DC3 are shown in black. (D) The expression trends of three gene clusters. The cashmere growth cycle is divided into three stages according to the expression patterns.

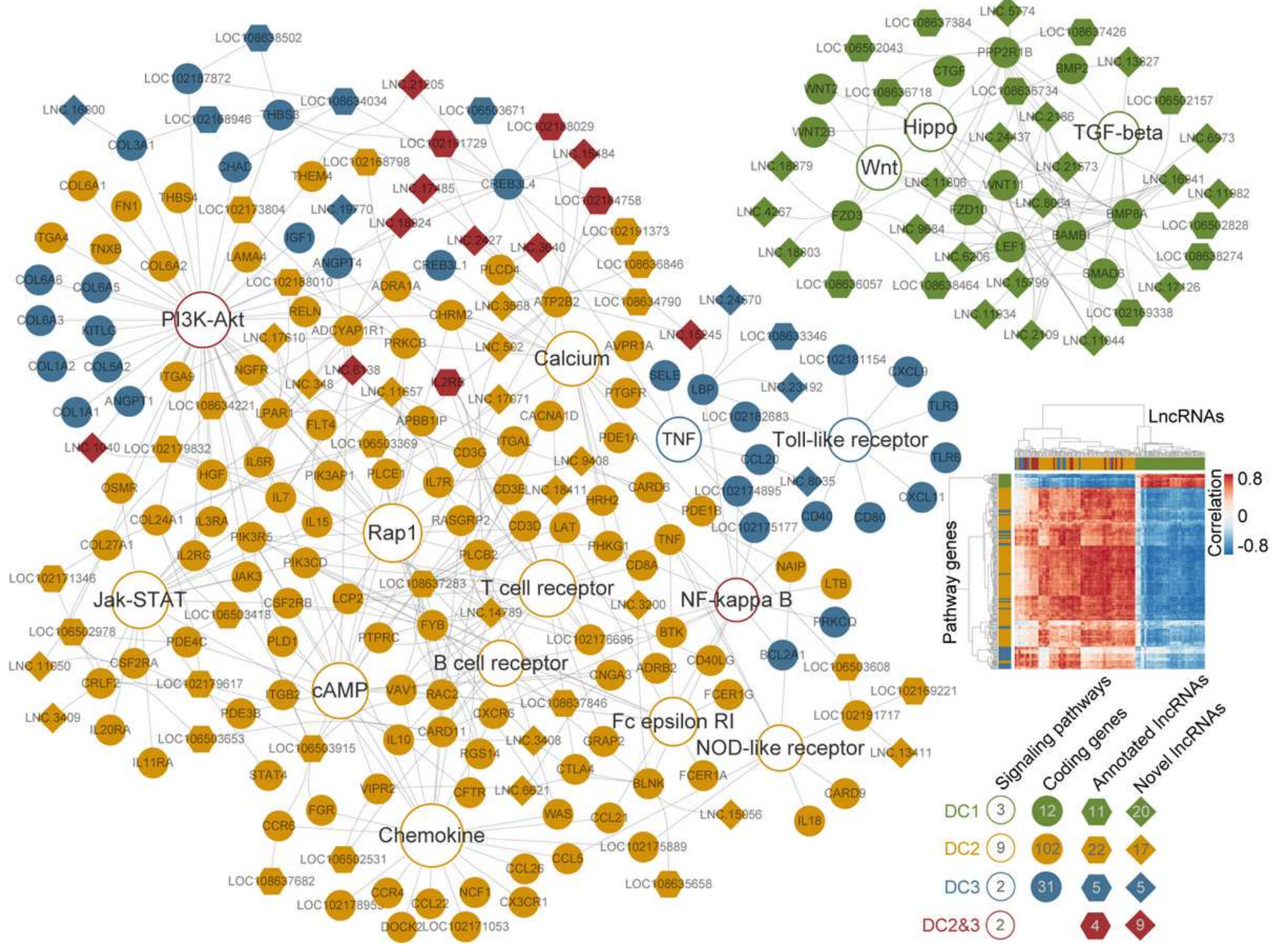

Figure 3

Pathway-mRNA-IncRNA co-expression network. A total of 16 signaling pathways, 143 pathway genes, and 93 co-expressed IncRNAs (Pearson correlation $\geq 0.8$ ) are involved in this network. The size of the pathway node is positively related to its degree. 
A

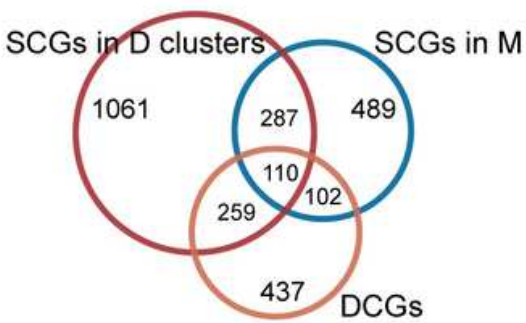

C
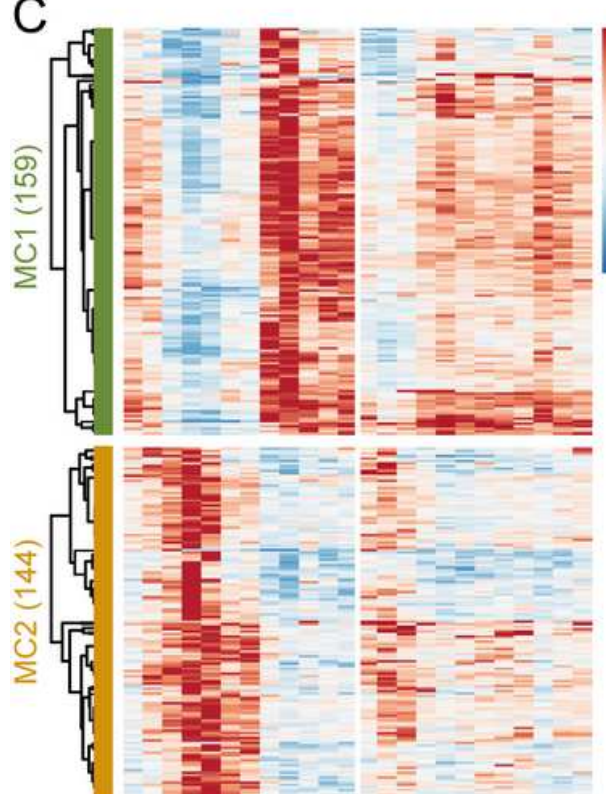

$\overbrace{}^{6} \sqrt{2}$

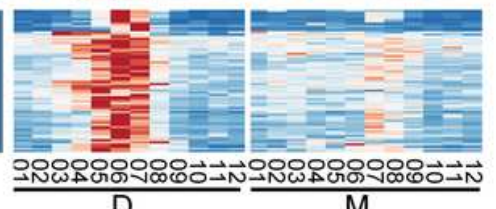

B

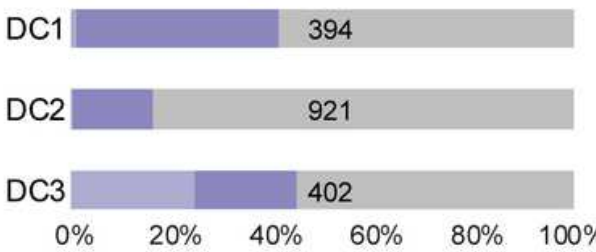

$\mathrm{D}$
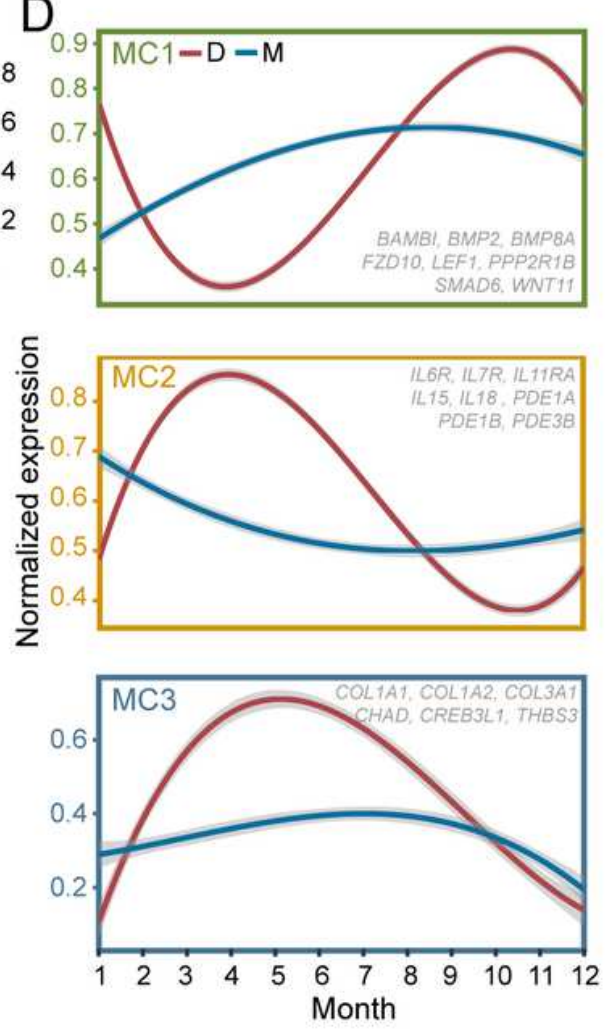

E $-\log 10(P$ value $)$
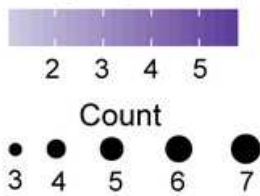

\begin{tabular}{ll|}
\hline & Hippo signaling pathway
\end{tabular}

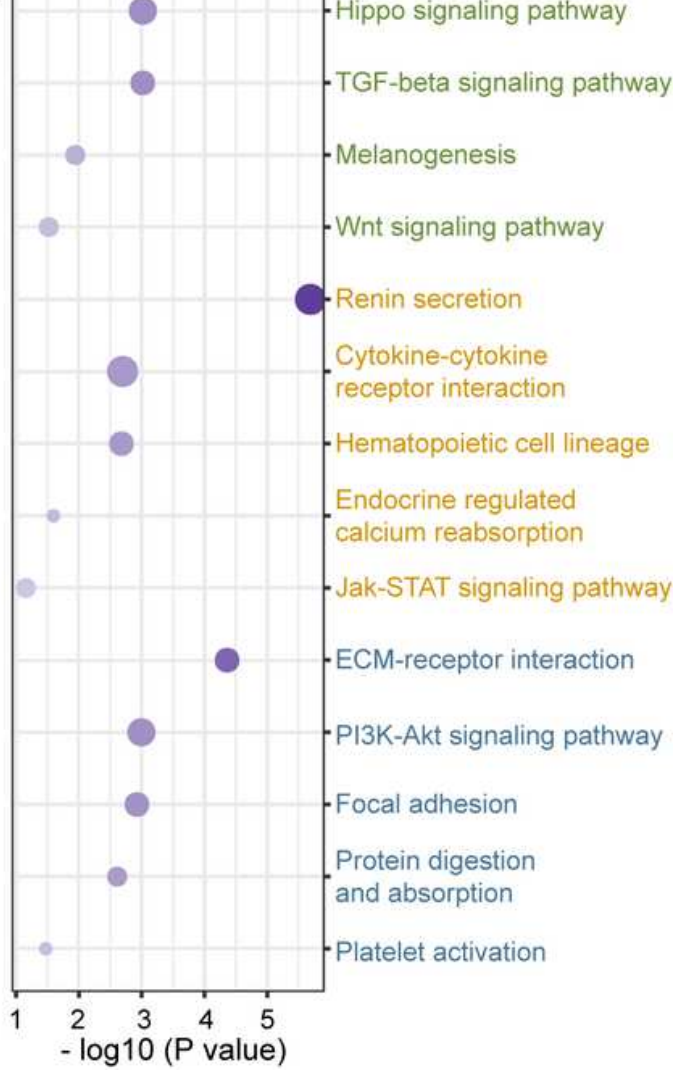

Figure 4

Analysis of differences in the expression patterns triggered by melatonin. (A) Venn diagrams showing SCGs in D clusters and M group and DCGs between D and M groups. (B) The changed and unchanged genes of three $D$ clusters after melatonin treatment. (C) Heatmap of the expression level of three $M$ clusters (MC1, MC2 and MC3) in D and M groups. (D) The expression patterns of three M clusters in D and $\mathrm{M}$ groups. (E) KEGG pathway enrichment of the genes in MC1, MC2 and MC3. 


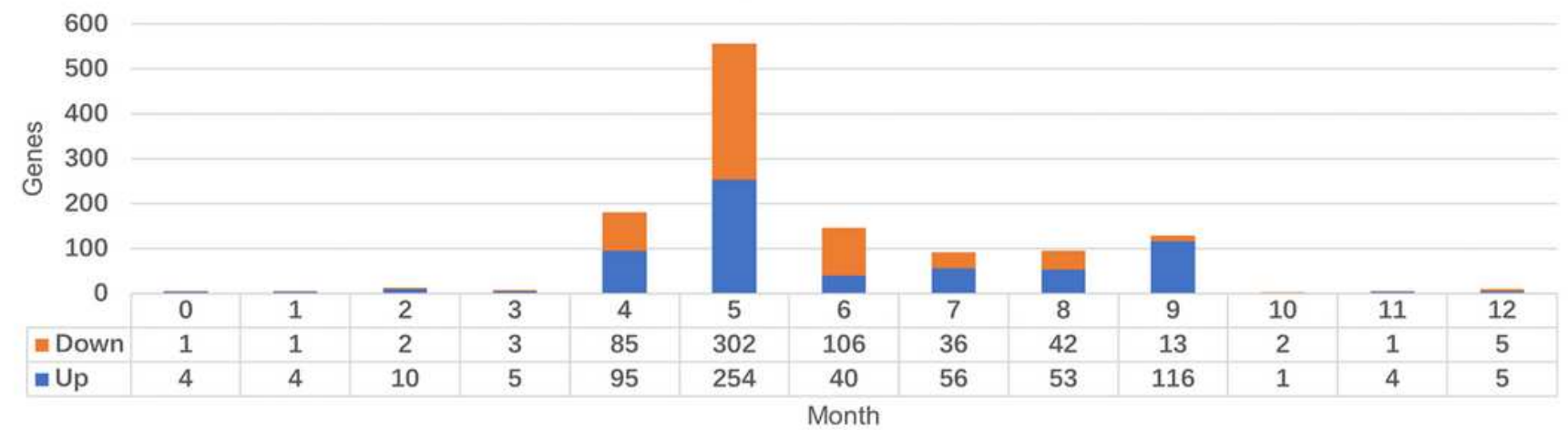

B

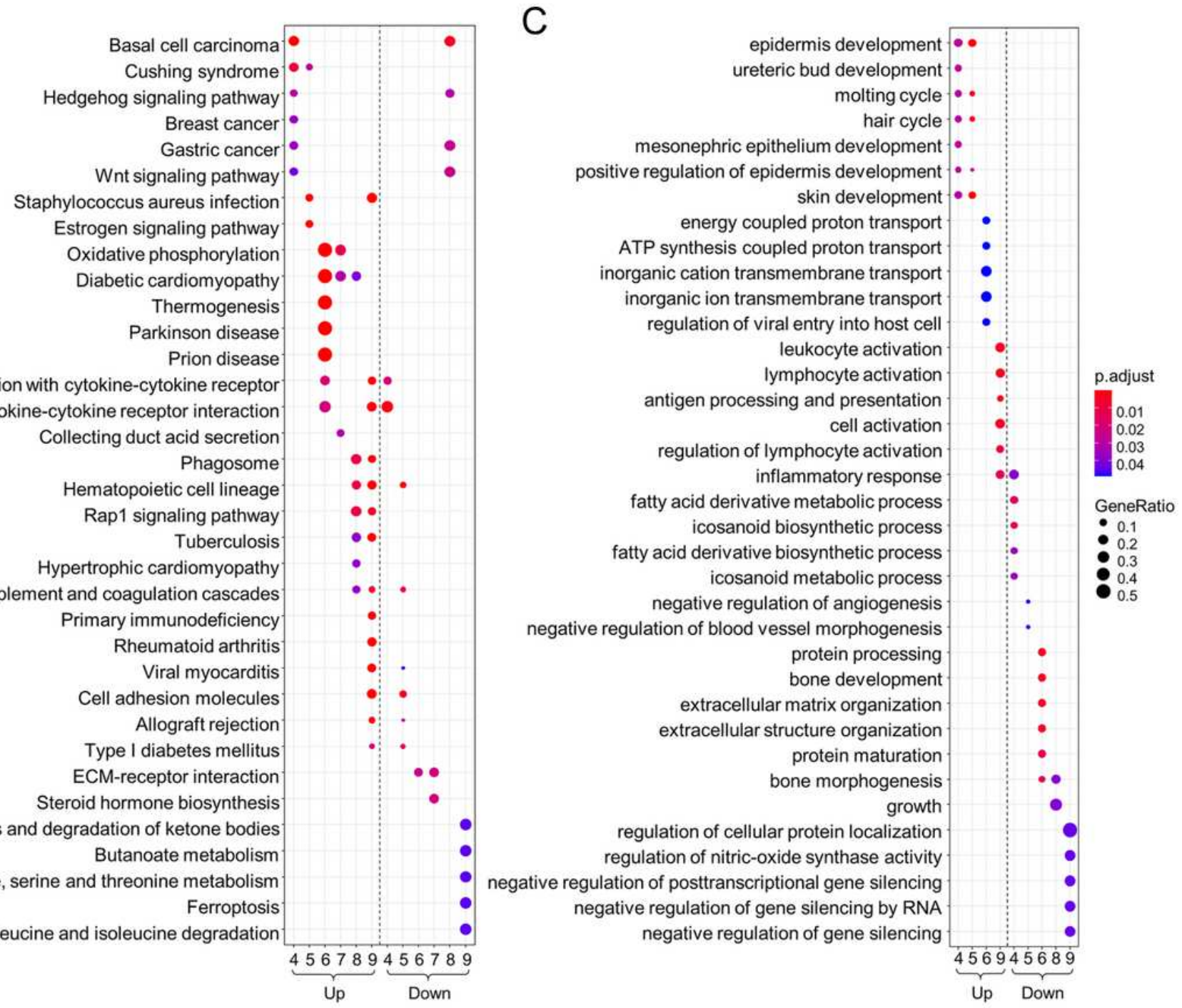

\section{Figure 5}

Detection of monthly differential expressed genes (DEGs) triggered by melatonin and pathway enrichment analysis. (A) The number and distribution of monthly differential expressed genes triggered by melatonin between M and D group. (B) The KEGG pathway enrichment analysis of monthly differential expressed genes. (C) The GO biological process enrichment analysis of monthly differential expressed genes. Note that up-regulated and down-regulated genes in different months are separated for GO \& KEGG analysis, in which 'Up' represents up-regulated genes, and 'Down' represents down-regulated genes. 
The color of nodes represents adjusted $p$ value, and the size of nodes represents the ratio of gene numbers.

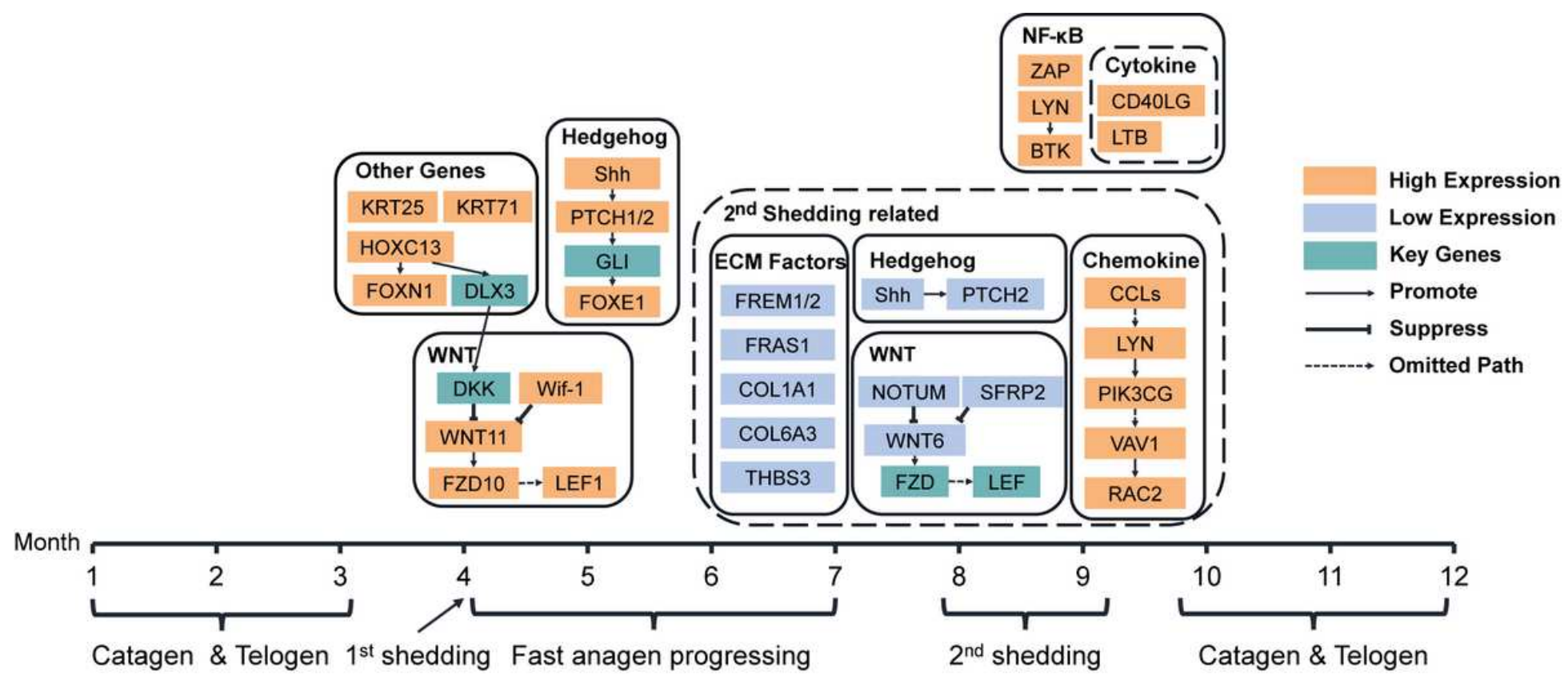

Figure 6

The putative pathway regulation model of the cashmere growth cycle triggered by melatonin. The signal pathways related to cashmere shedding are marked with dotted line boxes, and the other solid line boxes mark the signal pathways which may promote the hair follicle development.

\section{Supplementary Files}

This is a list of supplementary files associated with this preprint. Click to download.

- AdditionalFile1.csv

- AdditionalFile10.csv

- AdditionalFile11.csv

- AdditionalFile12.csv

- AdditionalFile13.docx

- AdditionalFile14.docx

- AdditionalFile2.csv

- AdditionalFile3.csv

- AdditionalFile4.csv

- AdditionalFile5.csv

- AdditionalFile6.csv

- AdditionalFile7.csv 
- AdditionalFile8.csv

- AdditionalFile9.csv 